\title{
OBJECT DETECTION USING WIRELESS SIGNALS
}

\author{
by
}

Gorla Praveen

A special study submitted in partial fulfillment of the requirements for the degree of Master of Engineering in Telecommunications

\author{
Examination Committee: Dr. Teerapat Sanguankotchakorn (Chairperson) \\ Dr. Poompat Saengudomlert (Member) \\ Dr. Attaphongse Taparugssanagorn (Member)
}

Nationality: Indian

Previous Degree: Bachelor of Engineering in Electronics and Communication Engineering

Jawaharlal Nehru Technological University, India

Scholarship Donor: AIT Fellowship

Asian Institute of Technology

School of Engineering and Technology

Thailand

September 2017 


\section{Acknowledgments}

I would like to express my profound gratitude to my esteemed advisor, Dr. Teerapat Sanguankotchakorn, Professor \& Head of Telecommunication Engineering field of study, for his advice throughout this course.

I would like to take this opportunity to thank Dr. Poompat Saengudomlert for his timely guidance on the report and Dr. Attaphongse Taparugssanagorn for his comments and suggestions on the report.

Finally, I thank my parents and friends for their support.

Gorla Praveen

November 2017 


\begin{abstract}
Wireless signals play major a role not only in establishing communication between the node to node but also for providing information about the other end of the destination node. Wireless signals especially used in radar engineering are used to detect the target eliminating the use of large number sensors.

RF wireless signals which are generated from the networking (WiFi routers) devices can be used for remotely detecting the object. And nowadays networking devices installed in the household and the companies are the part of modern communication and by using these devices for the above said would greatly reduce the use of sensors for object sensing. A person away from his home can detect the changes during his absence with the routers that are installed in his home.
\end{abstract}

This study aims at studying different techniques that are used for object detection using wireless signals at different scenarios. 


\section{Table of Contents}

\section{Chapter Title}

Page

Title Page

Acknowledgments

Abstract

Table of Contents

List of Figures

List of Tables

List of Abbreviations

List of Symbols

i

ii

iii

iv

$\mathrm{V}$

vii

viii

ix

Introduction

1.1 Overview

1.2 Problem Statement

1.3 Objectives

1.4 Limitations and Scope

1.5 Organization of the report

Literature Review

2.1 Radar systems

2.2 Bistatic Radar and Bistatic Range 3

2.3 Chebyshev filters

2.4 Kaiser-Bessel window

2.5 Algorithm 6

2.6 Some basic system models $\quad 7$

2.7 Wireless passive radar system models (s) 8

$\begin{array}{lll}2.8 & \text { Literature review of the research } & 18\end{array}$

2.9 Discussion 19

2.10 Software Validation $\quad 20$

3 Methodology 24

3.1 Power analysis $\quad 24$

3.2 Time delay analysis 38

4 Conclusion 44

$5 \quad$ References $\quad 45$

$7 \quad$ Appendices 46 


\section{List of Figures}

$\begin{array}{lll}\text { Figure Title } & \text { Page }\end{array}$

$2.1 \quad$ Block diagram of Bistatic radar 3

$2.2 \quad$ Bistatic range block diagram 4

$2.3 \quad$ Frequency response when filter type I order of four and $\epsilon=1 \quad 5$

$2.4 \quad$ Parametric family of Kaiser-window 6

2.5 CFAR by taking two adjacent cells around CUT 6

$\begin{array}{lll}2.6 & \text { Basic radar detection using bistatic range } & 7\end{array}$

2.7 Ambiguity diagram, Zero Doppler and range outs for DSSS/DBPSK Beacon signal $\quad 9$

2.8 Ambiguity diagram, Zero Doppler and range outs for DSSS/DQPSK Beacon signal. $\quad 10$

2.9 Geometrical representation of WiFi based passive radar. 11

2.10 Ambiguity function diagram when target $(12 \mathrm{~m})$ is moving to$\begin{array}{ll}\text { wards receiver with } 100 \mathrm{~ms} \text { time of reception } & 12\end{array}$

2.11 Ambiguity function diagram when two target $(35 \mathrm{~m}$ ) is moving towards receiver with $300 \mathrm{~ms}$ time of reception

2.12 Ambiguity function diagram when two target (12m )is moving towards receiver with $300 \mathrm{~ms}$ time of reception

2.13 Geometrical configuration for object detection in high clutter environment with bistatic baseline, bistatic range and bistatic angle was $7.6 \mathrm{~m}, 17.5 \mathrm{~m}$ and 460 .

2.14 Zero Doppler cut of the ambiguity function 16

2.15 Zero range cut of the ambiguity function 16

2.16 Doppler-range plot with target and bistatic range response 17

$\begin{array}{lll}2.17 & \text { Graphical representation of RSS vs CSI } & 18\end{array}$

2.18 Generation of Barker code, Spread signal (DSSS), DBPSK modulated signal and power Spectral density (fft) DSSS/DBPSK signal. 20

2.19 Ambiguity function - Doppler shift and delay of DSSS/DBPSK signal 21

2.20 Generation of Barher code, spread signal (DSSS), DBPSK modulated signal and power spectral density (fft) DSSS/DQPSK signal. 22

2.21 Ambiguity function - Doppler shift and dely of DSSS/DQPSK signal 23

3.1 Basic model using signal generator and spectrum analyser for feasibility test (Tx and Rx antennas of $2.4 \mathrm{GHz}$ are available in AIT TC Lab)

$\begin{array}{lll}3.2 & \text { when Tx-Rx are at } 3 \mathrm{~cm} \text { apart and when no obstacle is introduced } & 27\end{array}$

3.3 Received Peak value observation when Tx signal frequency is $2.4 \mathrm{GHz},-10 \mathrm{dBm} \quad 30$

$3.4 \quad$ Experimentation scenario 30 
3.5 Experiment 1: Graph comparing Rx amp dBm avg when obstacle is at different distances(LOS) b/w Tx and Rx, plotted for every $3 \mathrm{~cm}$ of move

3.6 Experiment 2: Graph comparing Rx amp dBm avg when obstacle is at different distances(LOS) b/w Tx and Rx, plotted for every $3 \mathrm{~cm}$ of move

3.7 Experiment 3: Graph comparing Rx amp dBm avg when obstacle is at different distances(LOS) b/w Tx and Rx, plotted for every $3 \mathrm{~cm}$ of move

3.8 Experimentation Scenario 36

$3.9 \quad$ System block diagram 38

3.10 GNU Radio Implementation 39

3.11 Target with delay of 1000 samples $\quad 40$

3.12 Target with delay of 500 samples 40

$3.13 \quad$ Target with delay of 250 samples 41

3.14 Target with delay of 100 samples 41

3.15 GNU Radio implementation for three target detection 42

3.16 Three target presence 42 


\section{List of Tables}

$\begin{array}{lll}\text { Table Title } & \text { Page }\end{array}$

2.1 Simulated and theoretical results of DSSS/DBPSK and DSSS/DQPSK signals. $\quad 10$

2.2 Experimental measurements are taken as mentioned 15

3.1 Rx and Tx values at $-10 \mathrm{dBm}$ Tx amplitude with varying frequency with $3 \mathrm{~cm}$ distance apart betwenw $\mathrm{Tx}$ and $\mathrm{Rx}$ when no obstacle is introduced

3.2 Receiver ( $\mathrm{Rx})$ observations recorded first (1st) time with distance between $\mathrm{Tx}$ and $\mathrm{Rx}$ as $30 \mathrm{~cm}$, no obstacle introduced

3.3 Receiver ( $\mathrm{Rx}$ ) observations recorded second (2nd) time with distance between Tx and $\mathrm{Rx}$ as $30 \mathrm{~cm}$, no obstacle introduced

$3.4 \quad$ Receiver ( $\mathrm{Rx})$ observations recorded third (3rd) time with distance between $\mathrm{Tx}$ and $\mathrm{Rx}$ as $30 \mathrm{~cm}$, no obstacle introduced

3.5 Receiver $(\mathrm{Rx}) \mathrm{dBm}$ recorded first (1st) time at various distance of obstacle position from the Tx

3.6 Receiver ( $\mathrm{Rx}) \mathrm{dBm}$ recorded second (2nd) time at various distance of obstacle position from the $\mathrm{Tx}$

3.7 Receiver ( $\mathrm{Rx}) \mathrm{dBm}$ recorded third (3rd) time at various distance of obstacle position from the Tx

3.9 Theoritical and simulated values 


\section{List of Abbreviations}

$\begin{array}{ll}\text { CFAR } & \text { Constant False Alarm Rate } \\ \text { CFR } & \text { Channel Frequency Response } \\ \text { CIR } & \text { Channel Impulse Response } \\ \text { CSI } & \text { Channel State Information } \\ \text { CSI } & \text { Channel State Information } \\ \text { DBPSK } & \text { Differential Binary Phase Shift Keying } \\ \text { DQPSK } & \text { Differential Quadrature Phase Shift Keying } \\ \text { DSI } & \text { Direct Signal Interference } \\ \text { DSSS } & \text { Direct Sequence Spread Spectrum } \\ \text { FMCW } & \text { Frequency Modulated Continuous Wave } \\ \text { GHz } & \text { Gigahertz } \\ \text { GPS } & \text { Global Positioning system } \\ \text { kHz } & \text { kilohertz } \\ \text { LOS } & \text { Line-of-Sight } \\ \text { MAC } & \text { Media Access Controller } \\ \text { MATLAB } & \text { Matrix Laboratory } \\ \text { MBPS } & \text { Megabytes Per Second } \\ \text { Mbps } & \text { Megabits per second } \\ \text { MHz } & \text { Megahertz } \\ \text { MSPS } & \text { Mega Samples Per Second } \\ \text { NLOS } & \text { Non-Line-of-Sight } \\ \text { OFDM } & \text { Orthogonal Frequency Division Multiplexing } \\ \text { PHY } & \text { Physical layer } \\ \text { PIR } & \text { Passive Infrared } \\ \text { PLCP } & \text { Physical Layer Convergence Protocol } \\ \text { PPDU } & \text { PLCP Protocol Data Unit } \\ \text { QAM } & \text { Quadrature Amplitude Modulation } \\ \text { RADAR } & \text { RAdio Direction And Ranging } \\ \text { RF } & \text { Radio Frequency } \\ \text { RSSI } & \text { Received Signal Strength Indicator } \\ \text { TOF } & \text { Time Of Flight } \\ \text { WiFi } & \text { Wireless Fidelity } \\ & \end{array}$




\section{List of Symbols}

$c \quad$ Velocity of light

$R x \quad$ Receiving antenna

$T x \quad$ Transmitting antenna

$V \quad$ Velocity of target

$\alpha \quad$ Non-negative arbitrary real number that determines Kaiser-Bessel window shape

$\chi \quad$ Basic ambiguity function

$\Delta T \quad$ Time of flight

$\tau \quad$ Time delay

$\mathrm{Br} \quad$ Bistatic range

$D_{r x} \quad$ Distance between $R_{x}$ and target

$D_{t x} \quad$ Distance between $T_{x}$ and target

$f_{b} \quad$ Beat frequency during FMCW analysis

$f_{D p} \quad$ Doppler Shift in frequency

$F_{r x} \quad$ Frequency of received signal

$F_{t x} \quad$ Frequency of transmitted signal

$G_{n}(w)$ Gain response of Chebyshev filter in dB

$I_{0} \quad$ Zeroth-order modified Bessel function

$s(t) \quad$ Represents DSSS modulated signal

$T_{m} \quad$ Time period of modulating signal in FMCW analysis

$w[n] \quad$ Represents response of Kaiser-Bessel window in $\mathrm{dB}$

B Bandwidthg of a signal

f Frequency of a signal

L Distance between transmitter and receiver

$\mathrm{R} \quad$ Range of object from the system during FMCW analysis

$\mathrm{T}$ Time period of observation during low clutter analysis 


\section{Chapter 1}

\section{Introduction}

\subsection{Overview}

Wireless communications had paved way for establishing communication from place to place and from node to node with data rates ranging from bits per seconds to gigabytes per second. It has not only been used as a means of communication but also for localization of things. Localization by using wireless signals is the most advanced and promising technology in this era of the Internet of Things which can be used for detecting the objects within the shortest range but with the most accuracy. In general, Global Positioning System (GPS) helps in tracking the objects only in outdoor positioning with a meter level accuracy and for indoor positioning of objects we need a varied type of sensors that to be employed for tracking the object moments. When sensors such as Ultrasonic and PIR are used there is a great probability of occurrence of an error while tracking multiple objects when there is a presence of multiple bodies. These sensors fail in making an optimal decision in distinguishing the direction of motion of multiple objects. When the accuracy is the most priority then a large number of sensors can be deployed but it increases the bulkiness with only a little improvisation of accuracy. As wireless signals are prone to interference many digital signal processing techniques can be used to reduce their effect increasing the efficiency of detection. However, Indoor positioning using wireless signals is the promising one for localization in this era of the Internet of Things. A lot of research from the past to the present has been done on Wireless Indoor localization by many scholars, in which their work will be discussed in detail in the later stages of this report.

\subsection{Problem Statement}

Indoor Localization in this era of the Internet of Things has been in raise to detect the objects with most accuracy. But it greatly needs a large number of sensors and many networking devices to be employed for object sensing with a direction of motion and connecting them to the rest of the world.

\subsection{Objectives}

- To study about the techniques of object detection using Wireless signals.

- To study on improving the accuracy of detecting objections when WiFi( $802.11 \mathrm{~b} / \mathrm{g} / \mathrm{n} / \mathrm{ac})$ and FMCW signals are used.

- To compare different methods that work in different environments with accuracy and cost as major criteria. 


\subsection{Limitations and Scope}

These systems are capable of detecting the objects in various environments but these lack portability to use for regular use and the cost of the system is very expensive.F Further scope is to develop these scenarios in single board computers.

\subsection{Organization of the report}

To compare different methods that work in different environments with accuracy and cost as a major criterion.

Chapter 1: Introduction: Overview, problem statement, objective, scope and limitations, and organization of the report.

Chapter 2: Literature review on the current state of research.

Chapter 3: Basic research methodologies that are used in localization from the past.

Chapter 4: Basic experimentation for Object detection.

Chapter 5: Conclusions 


\section{Chapter 2}

\section{Literature Review}

This chapter includes basic concepts evolved during the course of research from the past to the present on Indoor localization for object detection with certain parameters like the distance between the object(s) from the reference point, the velocity of an object, direction and the phase of motion of an object. This also includes an explanation of some digital processing techniques and algorithms which are used for effective analysis of signal at the receiver.

\subsection{Radar systems}

Radar systems consist of transmitters and receivers for object tracking in which these components are used for calculating Velocity, distance from a reference point, angle of arrival and time of arrival of the object. Generally, these systems are large in size and find its applications for military purposes but nowadays tiny radar systems are designed for native purpose using available sensors and wireless signals of unlicensed band.

\subsection{Bistatic Radar and Bistatic Range}

Bistatic radar as shown in Figure 2.1, is a system having a transmitter and the receiver. The separation between them is almost correspondingly comparable to the approximated target range. In opposite to this, there are other radar systems, where these are different from Bistatic radar in a way of separate $\mathrm{Tx} \mathrm{Rx}$, the range of detection, the principle of detection, type and number of antennas used.

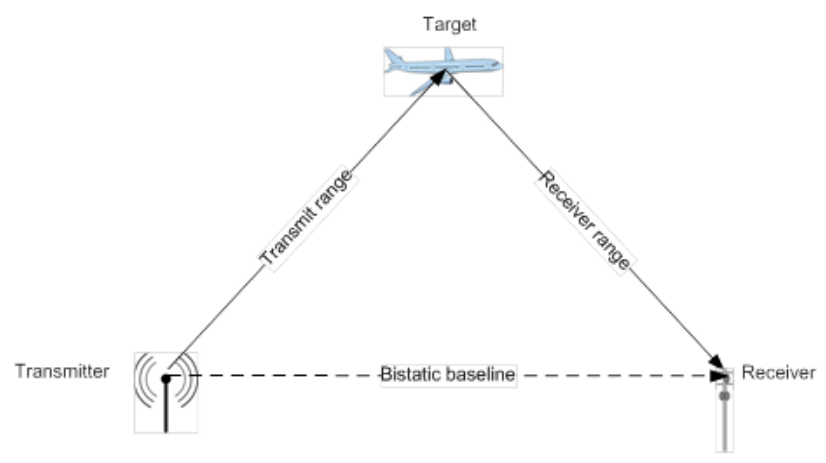

Figure 2.1: Block diagram of Bistatic radar

(NickYoung.NATS, 2017) 
Basic determination of range using separate transmitter and receiver by calculating time of arrival of the object is called as Bistatic range. Bistatic range as shown in Figure 2.2 is equivalent to $D_{t x}+D_{r x}-L$ where $D_{t x}, D_{r x}$ and $L$ are distance between transmitter target object, target object receiver and transmitter receiver respectively.

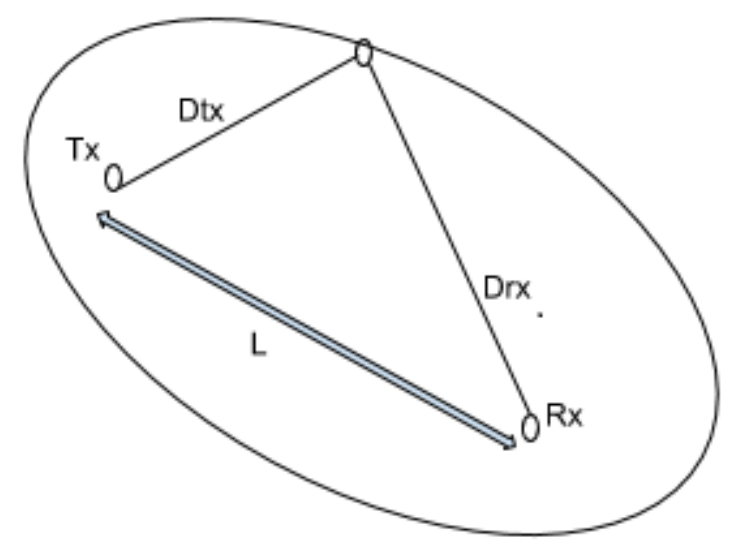

Figure 2.2: Bistatic range block diagram

(Willis, Nicholas.2007.BistaticRadar)

\subsection{Chebyshev filters}

As wireless communications are more prone to noise and interference, filters are used to reduce their effect in the final output of the received signal. Chebyshev filters are used for minimizing the error between the idealized filter and the realized filter over the particular range. Generally, these characteristics are mathematical and inherited from Chebyshev polynomials. As these filters are passband ripple they show the smooth response for passband and irregular response in the stop band.

The gain response $G_{n}(\omega)$ as shown in figure 2.3 of Chebyshev is most commonly used filter of Type I which is represented in (2.1).

$$
G_{n}(\omega)=|H+n(j \omega)|=\frac{1}{\sqrt{1+\epsilon^{2} T_{n}^{2}\left(\frac{w}{w_{0}}\right)}}
$$




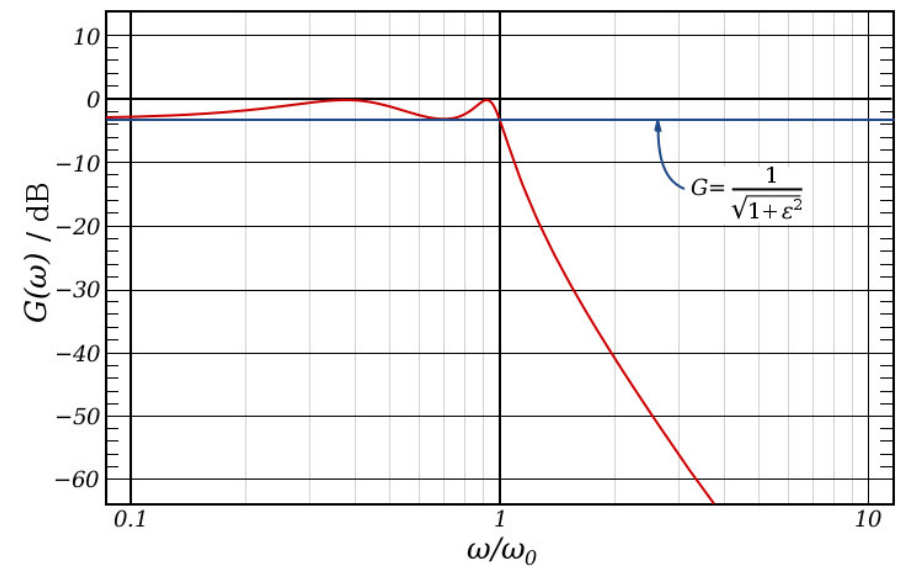

Figure 2.3: Frequency response when filter type I order of four and $\epsilon=1$

(Willams, B., et al. (2006). Electronic Filter Design Handbook)

\subsection{Kaiser-Bessel window}

Kaiser window (Kaiser-Bessel window ) as shown in figure 2.4 is one of the window filter used for processing the signal in digital signal processing. This is defined as follows.

$$
\begin{aligned}
w[n] & =\frac{I_{0}\left(\left(\beta \sqrt{1-\left(\frac{2 n}{N-1}-1\right)}\right)\right.}{I_{0}(\Pi \beta)}, 0 \leq n \leq N-1 \\
& =0, \text { otherwise. }
\end{aligned}
$$

Where in (2.2) $N$ is the length of the sequence in odd numbers, $I_{o}$ is the zeroth-order modified Bessel function of the first kind, $\beta=\pi \alpha$ and $\alpha$ is an arbitrary and non-negative real number that determines the shape of the window. 


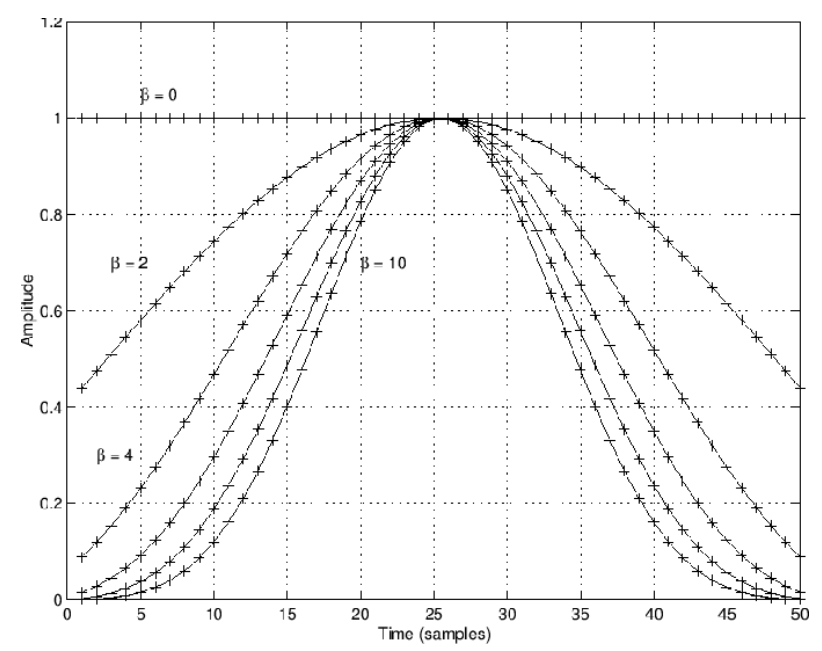

Figure 2.4: Parametric family of Kaiser-window

(Smith III. (2011) Spectral Audio Signal Processsing: CCRMA)

\subsection{Algorithm}

\section{Constant False Alarm Rate (CFAR) and CLEAN algorithm}

CFAR is an adaptive algorithm for detecting the target object by calculating the threshold value of the power at the receiver. When the received power level is above the threshold then it is considered to be originated from the target and the object is detected. As the threshold level decreases then there is a possibility of detecting more targets with an increase in a number of false alarm rate. In general, a threshold is calculated by cell- averaging method by taking a set of block of cells around the cell under test (CUT) as shown in figure 2.5and calculating the average of the power.

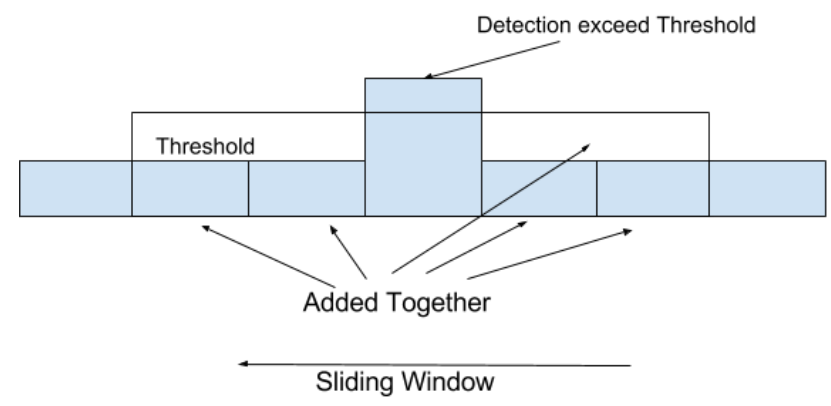

Figure 2.5: CFAR by taking two adjacent cells around CUT

CLEAN is a computational algorithm used for deconvolution of images by iteratively considering the highest point value of the image and subtracting it from the small gain of this point source system 
impulse function until the highest point value is less than the desired threshold.

\subsection{Some basic system models}

Here we use concept of bistatic range, basic concept used in radar systems as shown in figure 2.6 in deducing the target range.

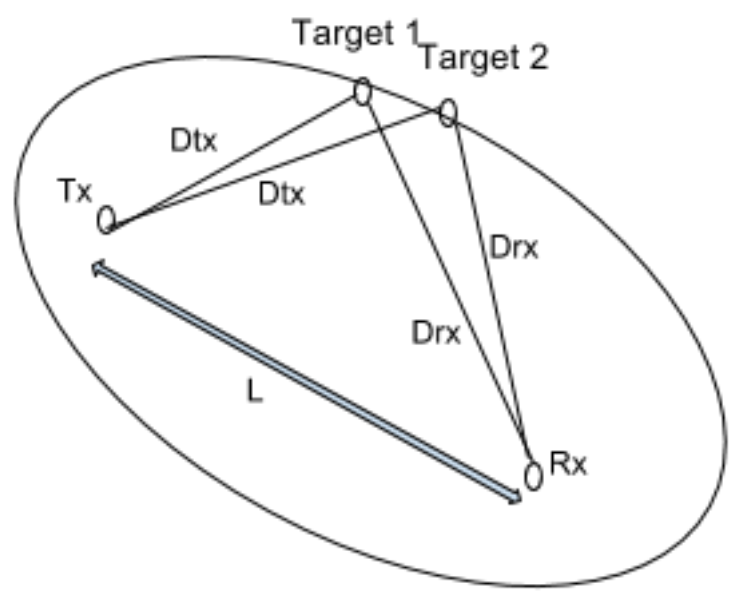

Figure 2.6: Basic radar detection using bistatic range

(Willis, Nicholas. 2007. BistaticRadar)

From the figure 2.6, sum of distances of $D_{r x}$ and $D_{t x}$ remains same when the object is detected on the ellipse where $D_{t x}$ and $\operatorname{Dr} x$ are the distance of target from Tx and RX respectively. Here $L$ is the separation between the Tx and RX

When transmitter generates the signal with a frequency of $F_{t x}$, then it gets reflected by object with a frequency of $F_{r x}$ and it is processed by the receiver.

$$
\begin{gathered}
F_{t x}-F_{r x}=\frac{1}{\Delta T} \\
D=\frac{c}{\Delta T} ;
\end{gathered}
$$

Where $c$ in (2.4) is the speed of light and $D$ is almost equal to the sum of $D_{r x}$ and $D_{t x}$

$$
D \approx D_{t x}+D_{r x}
$$

Hence from (2.4) the target range which is equals to bistatic range $B_{r}$ is represented in (2.6)

$$
B_{r}=D-L
$$




\subsection{Wireless passive radar system models (s)}

In passive radar-based detection, there is no dedicated transmitter (Tx), instead, receiver (Rx) uses some third party transmitted data from available transmitter(Tx) by estimating the arrival time.

\section{Low clutter environment}

The first experiment to detect humans using wifi signal was done by Guo et al.,2008 Using WiFI Beacon transmission for wireless based passive radar in low clutter environment[1].Beacon signals as one frame which is emitted periodically by the transmitter can be used to detect the presence of objects. Emitted signals consist of two parts (DBPSK coding with 1 Mbps and DQPSK coding with $2 \mathrm{Mbps}$ ) is modulated by DSSS modulation with a barker code of eleven elements.

Barker code eleven element series : $[+1,-1,+1,+1,-1,+1,+1,+1,-1,-1,-1]$

General equation of DSSS modulation is represented in (2.7)

$$
s(t)=\exp \left(j 2 \pi f_{0} t\right) \sum_{n}^{N} \exp (j \psi) p(t)
$$

Where $n$ is number of bits and $p(t)$ is the pulse strain function.

The below (2.8), (2.9), (2.10) represents the 1 Mbps DSSS DQPSK, 2 Mbps DSSS DQPSK and rectangular pulse respectively.

$$
\begin{gathered}
s(t)=\exp \left(j 2 \pi f_{0} t\right) \sum_{n=0}^{N-1} \sum_{m=0}^{M-1} \exp \left(j \psi_{n, m}\right) p(t-m T-11 n T) \\
s(t)=\exp \left(j 2 \pi f_{0} t\right) \sum_{n=0}^{(N-1) / 2} \sum_{m=0}^{M-1} \exp \left(j \psi_{n, m}\right) p(t-m T-22 n T) \\
p(t)=1,0 \leq t \leq T \\
=0, \text { otherwise }
\end{gathered}
$$

Ambiguity function analysis is done to verify the received signal and the amount of distortion, 11 MHz [1] signal bandwidth is used in simulating in Matlab. The basic ambiguity function is expressed in $(2.11)$. 


$$
\left|\chi\left(\tau, f_{D p}\right)\right|=\left|\int_{-\infty}^{\infty} x(t) x(t-\tau) \exp \left(2 \pi f_{D p} t\right) d t\right|
$$

Substituting (2.8) and (2.9) in (2.11) we can obtain DSSS DBPSK and DSSS DQPSK ambiguity functions respectively. Upon theoretical calculation and the simulation results from Guoetal., 2008 as shown in figure 2.7 and figure 2.8 which are represented in table 2.1
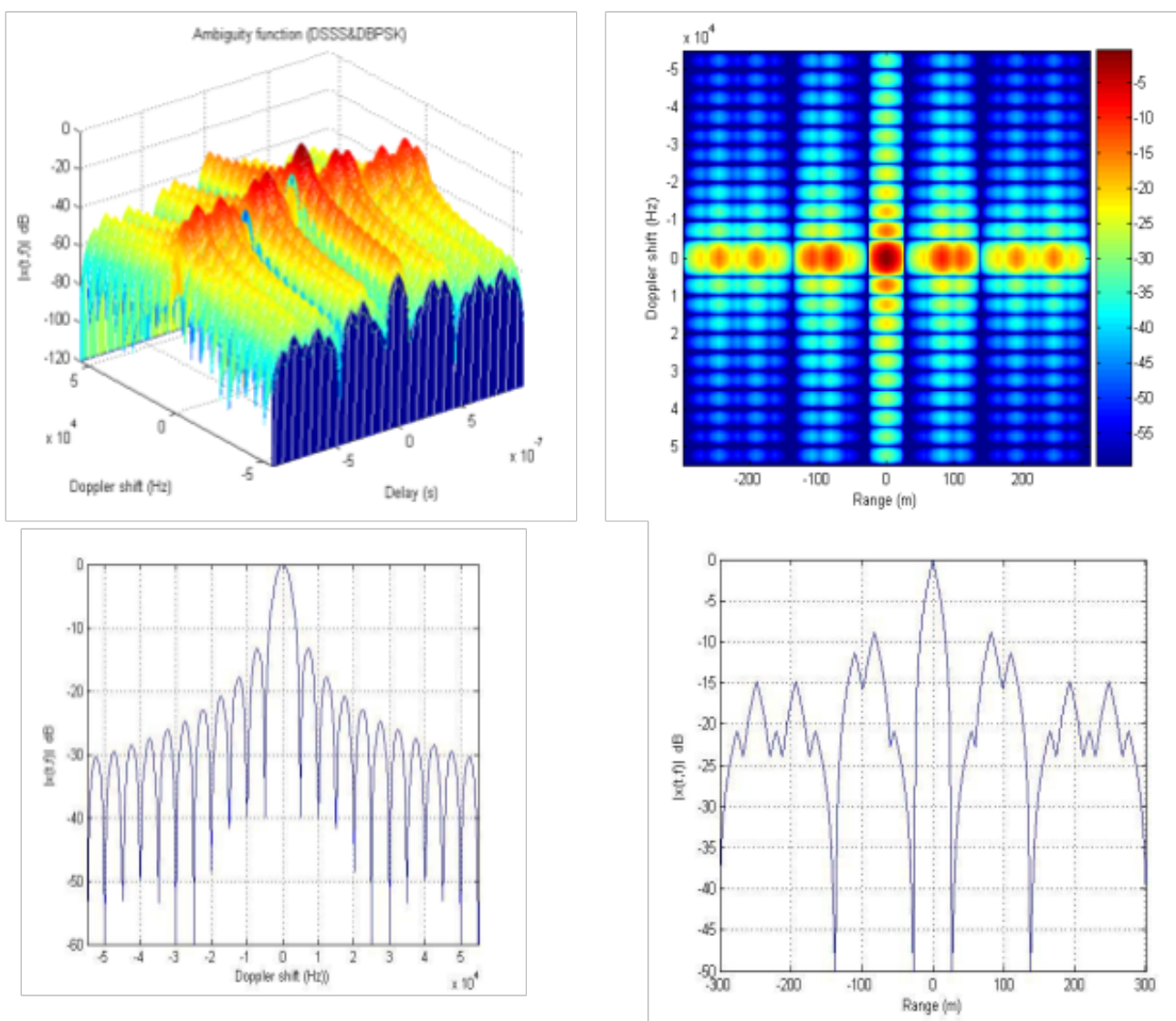

Figure 2.7: Ambiguity diagram, Zero Doppler and range outs for DSSS/DBPSK Beacon signal

(Guo et al., 2008) 

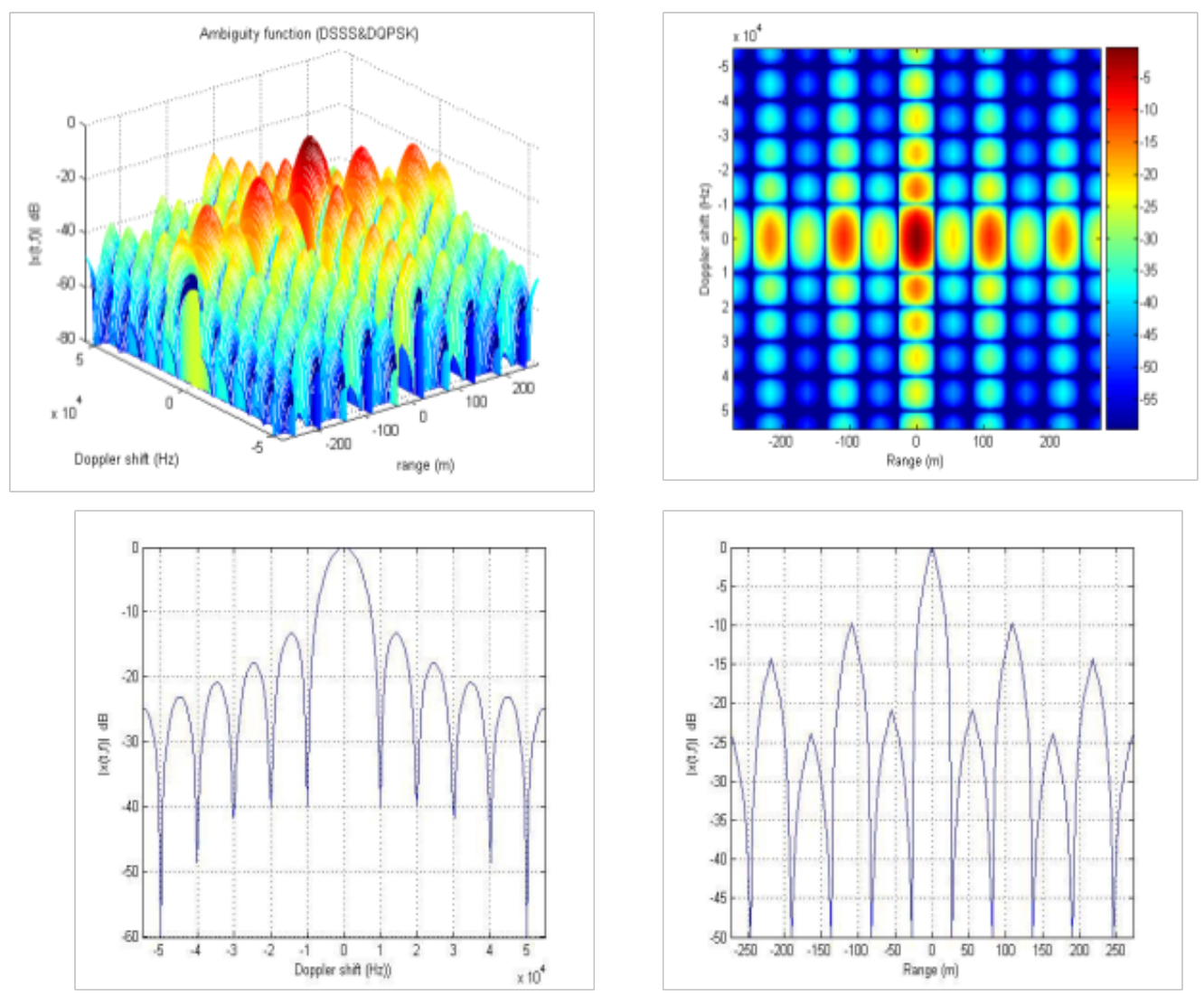

Figure 2.8: Ambiguity diagram, Zero Doppler and range outs for DSSS/DQPSK Beacon signal.

(Guo et al., 2008)

\begin{tabular}{|l|l|l|l|}
\hline Modulation & Resolution & Simulation & Theory \\
\hline DSSS/DBPSK & Range & $15.9 \mathrm{~m}$ & 13.6 \\
\hline DSSS/DBPSK & Doppler & $4.61 \mathrm{kHZ}$ & $5.2 \mathrm{kHZ}$ \\
\hline DSSS/DBPSK & Range & $15.9 \mathrm{~m}$ & $13.6 \mathrm{~m}$ \\
\hline DSSS/DBPSK & Doppler & $9.21 \mathrm{kHz}$ & $10.4 \mathrm{kHZ}$ \\
\hline
\end{tabular}

Table 2.1: Simulated and theoretical results of DSSS/DBPSK and DSSS/DQPSK signals.

(Guo et al., 2008) 


\section{Practical implementation}

Following conclusions from theoretical ambiguity function analysis in low clutter region. Experimentation is done in Guo et al., 2008 by using WiFi transmitter (DWL-200AP) with 2.437 central frequency having $20 \mathrm{dBm}$ peak EIRP theoretical assumption with a transmitting antenna gain of $5 \mathrm{dbi}$ and zero loss.

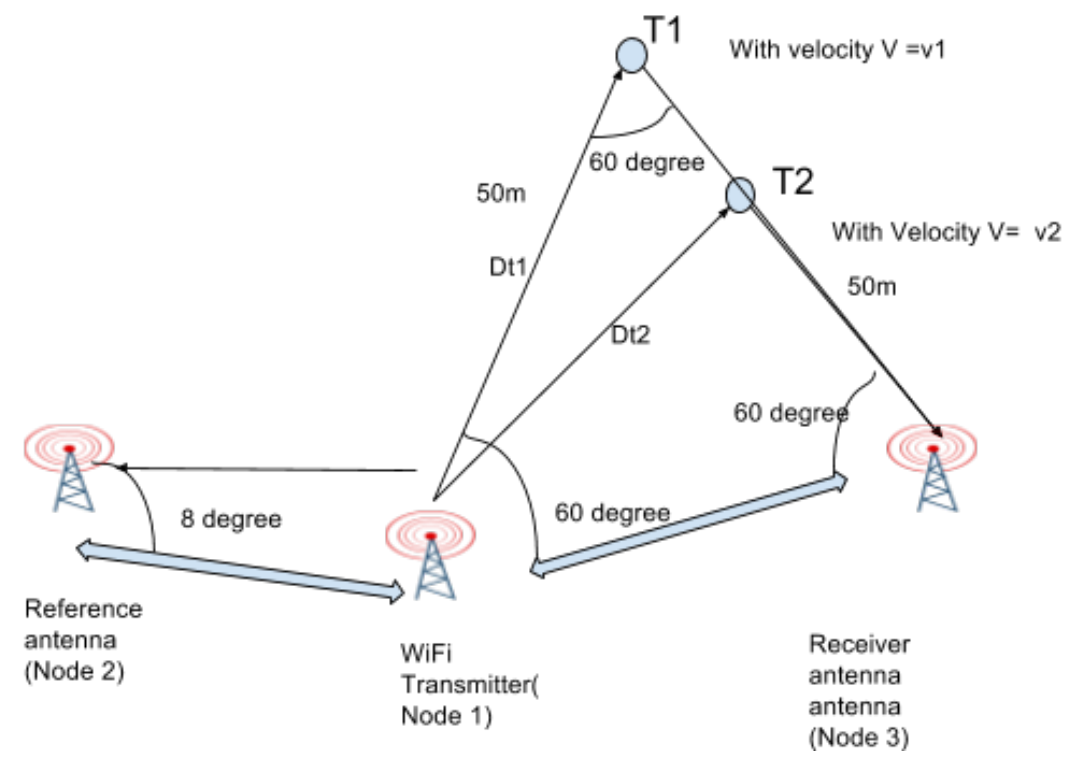

Figure 2.9: Geometrical representation of WiFi based passive radar.

(Guo et al., 2008)

Experimentation is conducted in Guoetal., 2008 using the configuration represented in the figure 2.9 with two antennas having narrow beam width of $24 \mathrm{dBi}$ gain. Received signal is processed in Matlab with $100 \mathrm{MHz}$ sampling frequency using NetRad in [1] by calculating doppler resolution in the equation (2.12) and using (2.11) the following results shown in figures $2.10,2.11$ and 2.12 are obtained.

$$
\Delta f=\frac{1}{T}<f_{d p}=\frac{V \cos \frac{\beta_{i}}{2} \cos \left(\alpha_{i}\right)}{\frac{c}{f}}
$$

Where $\Delta f$ is doppler resolution, $f_{D p}$ is doppler shift, $T$ is period of observation, $V$ target velocity, $c$ is speed of light, $f$ is transmitter frequency which is $2.4 \mathrm{Ghz}, \beta_{i}=60 \mathrm{deg}$ and $\alpha_{i}$ is $30 \mathrm{deg}$ from figure 2.9 .

Transmitting antenna which transmits Beacon signal is initiated, first a single target at a distance of $12 \mathrm{~m}$ from the receiver is made to move towards the receiver with $100 \mathrm{~ms}$ reception span. Figure 2.10 shows the target detection range. 


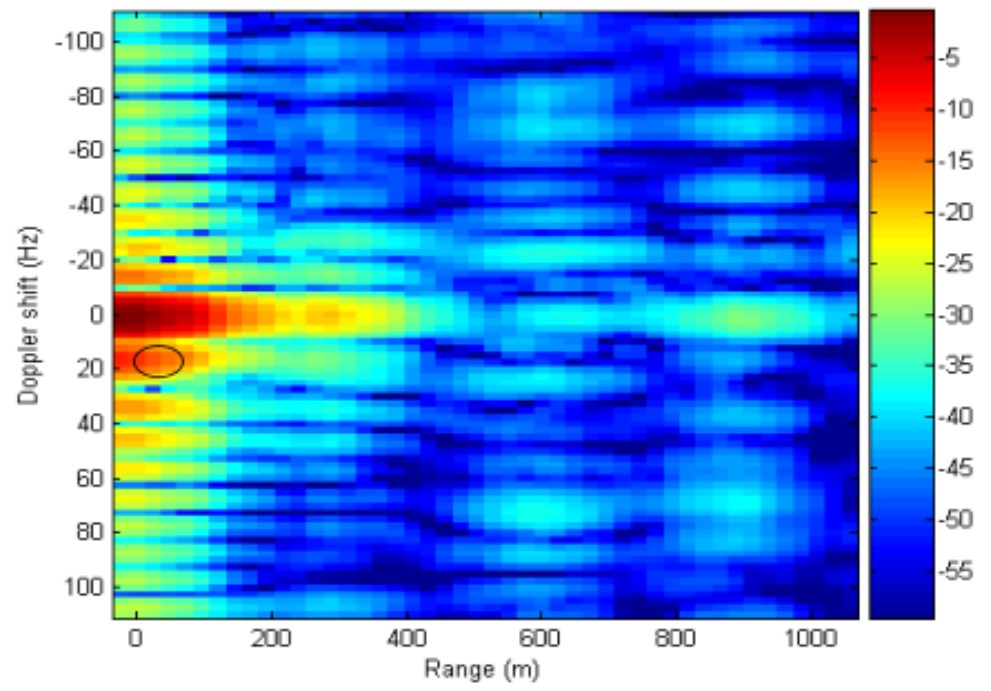

Figure 2.10: Ambiguity function diagram when target $(12 \mathrm{~m})$ is moving towards receiver with $100 \mathrm{~ms}$ time of reception

(Guo et al., 2008)

As there are many side lobe from the figure 2.10 and to reduce them the time reception is increased to $300 \mathrm{~ms}$ and the following results as shown in figures 2.11 and 2.12are obtained when two targets are at near( $12 \mathrm{~m}$ from receiver) and far (35 $\mathrm{m}$ from receiver) distances .

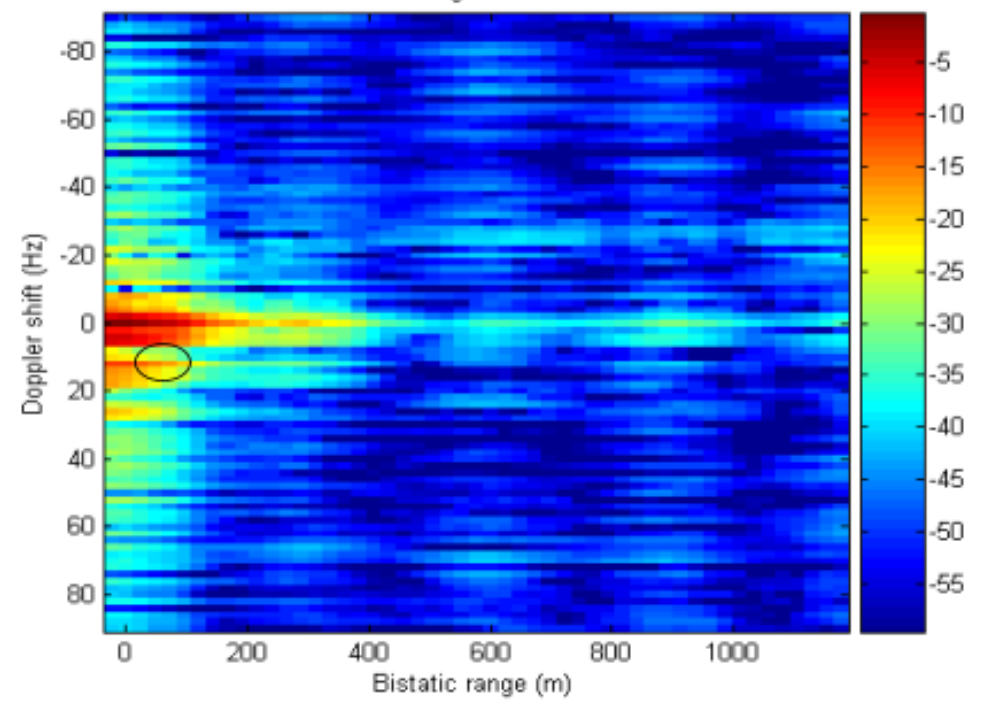

Figure 2.11: Ambiguity function diagram when two target (35m )is moving towards receiver with $300 \mathrm{~ms}$ time of reception

(Guo et al., 2008) 


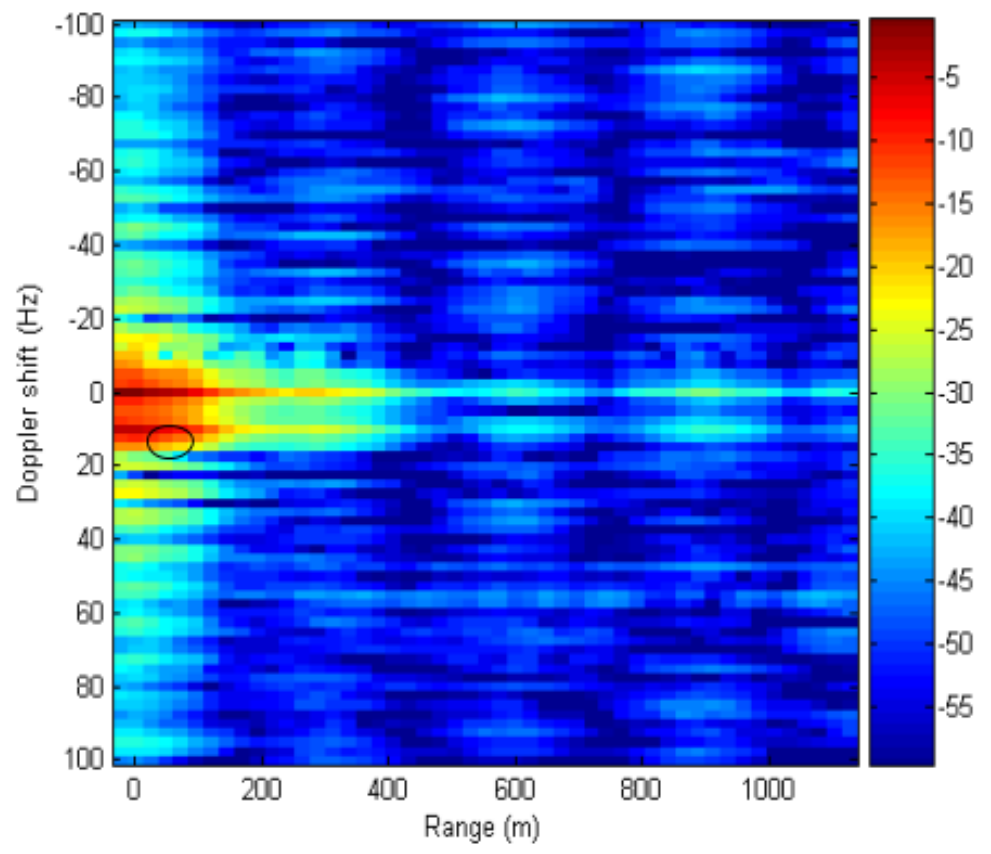

Figure 2.12: Ambiguity function diagram when two target $(12 \mathrm{~m})$ is moving towards receiver with $300 \mathrm{~ms}$ time of reception

(Guo et al., 2008)

So the above experimentation Guo et al., 2008 show that 802.11 Beacon signals can be used for detection of targets using WLAN. But this works in an low clutter environment where the desired results may vary when done in high clutter environment.

\section{High clutter environment}

In section 2.7 of low clutter we discussed target detection in low clutter environment but when comes to high clutter environment where walls, floors, concrete buildings are the instruments of noise and interference which results in an error in detection. So we employ digital processing techniques for reducing the noise and error in detecting the targets.

WiFi transmitter simulated with 64- QAM OFDM signal is used to transmit the signal at $54 \mathrm{Mbps}$ data rate with $16 \mathrm{Mhz}$ bandwidth in [2] by Chetty et al., and the configuration of antennas is shown in the figure 2.13 . 


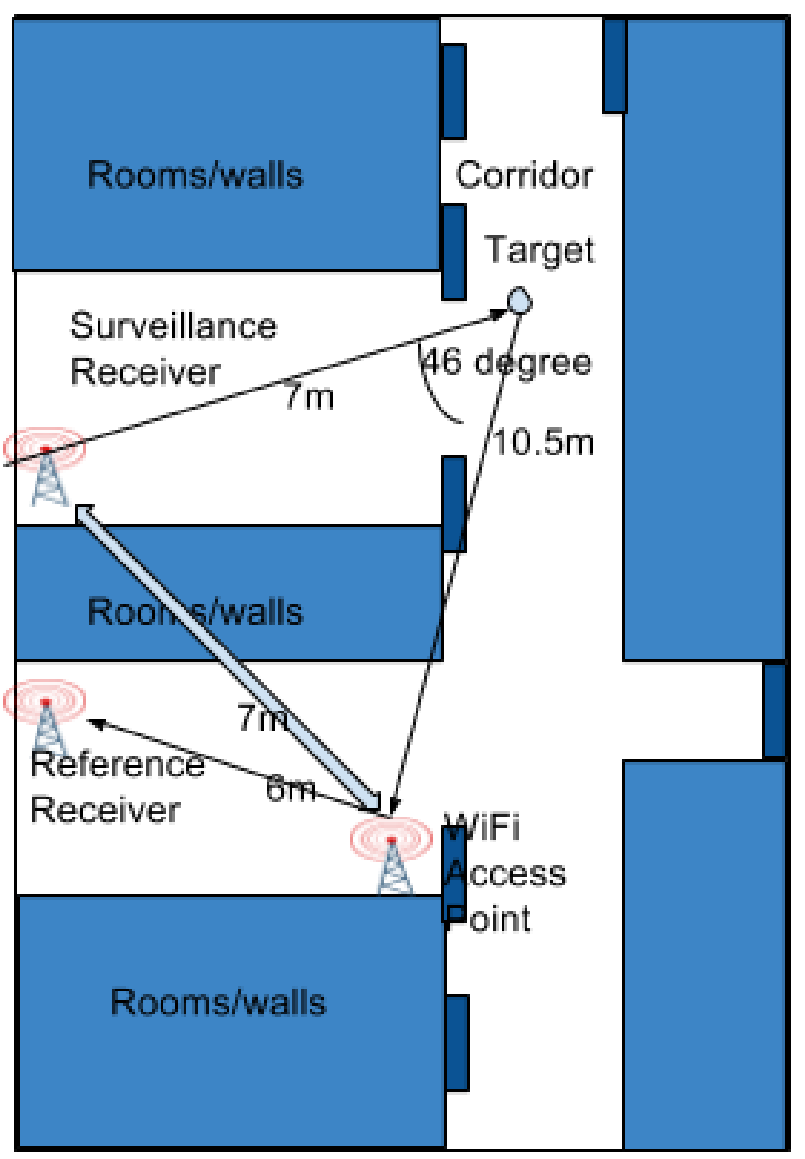

Figure 2.13: Geometrical configuration for object detection in high clutter environment with bistatic baseline, bistatic range and bistatic angle was $7.6 \mathrm{~m}, 17.5 \mathrm{~m}$ and 460 .

(Chetty et al., 2009)

As this experiment is done in high clutter region then the received signal is prone to noise, Here in Table 2.2 represents the experimental measurements of system noise and the clutter response by Chetty et al 


\begin{tabular}{|l|l|l|l|}
\hline Test & Target & Target Pace & Notes \\
\hline $\begin{array}{l}\text { 1[System noise and clutter re- } \\
\text { sponse] }\end{array}$ & No & - & $\begin{array}{l}\text { Noise measurement with no } \\
\text { antenna }\end{array}$ \\
\hline $\begin{array}{l}\text { 2[System noise and clutter re- } \\
\text { sponse] }\end{array}$ & No & - & $\begin{array}{l}\text { Noise measurement of an- } \\
\text { tenna connected }\end{array}$ \\
\hline $\begin{array}{l}\text { 3[System noise and clutter re- } \\
\text { sponse] }\end{array}$ & No & - & $\begin{array}{l}\text { Antenna connected with idle } \\
\text { WLAN }\end{array}$ \\
\hline $\begin{array}{l}\text { 4[System noise and clutter re- } \\
\text { sponse] }\end{array}$ & No & - & Antenna connected with Data \\
transfer by WLAN
\end{tabular}

Table 2.2: Experimental measurements are taken as mentioned

(Chetty et al., 2009)

Ambiguity function using (2.11) is measured in Chetty et al., 2009 for a time period of about 100ms is analysed and the simulated results are presented in the figures 2.14 and 2.15 . It is observed from both the figures that the bistatic range resolution is $14 \mathrm{~m}$ with $-19 \mathrm{db}$ of sidelobes of highest peak from the mainlobe and the resolution of $8 \mathrm{~Hz}$ with $-8 \mathrm{db}$ of sidelobes of highest peak from the main lobe. 


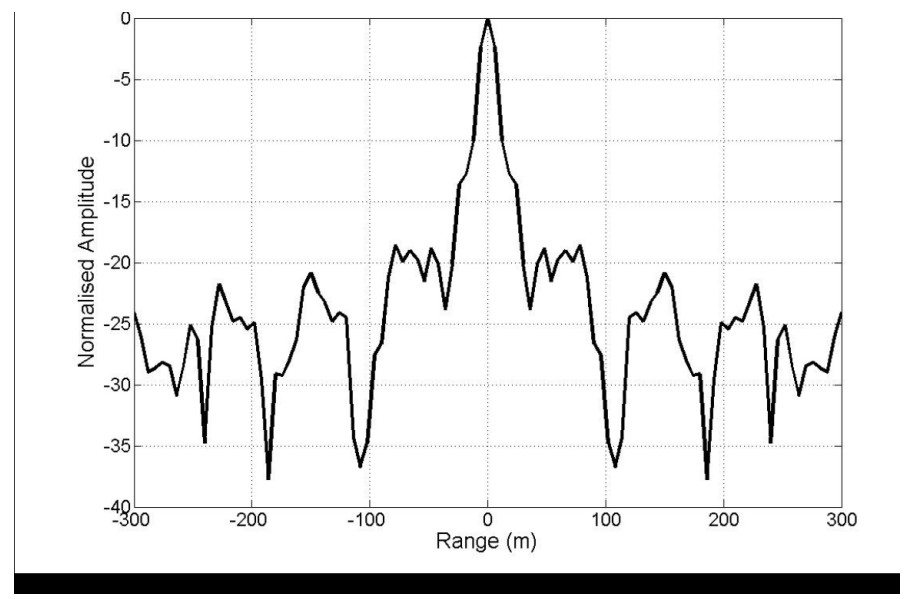

Figure 2.14: Zero Doppler cut of the ambiguity function

( Chetty et al., 2009)

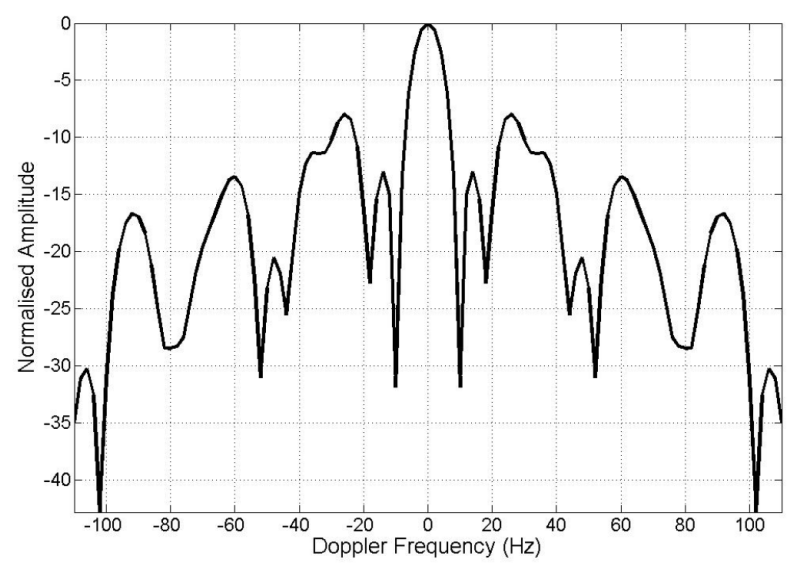

Figure 2.15: Zero range cut of the ambiguity function

( Chetty et al., 2009)

After analyzing the cross ambiguity function, a test is conducted when there is no presence of target using measurements from table 2.2(1-4). This intention is to cancel DSI (Direct Signal Interference) using the self-ambiguity from the cross ambiguity function when target detection data is received. Then again experimentation is done in Chetty et al., using measurements from table 2.2 (9-10) with figure 2.16 representing Doppler range plots when there is single person presence. 


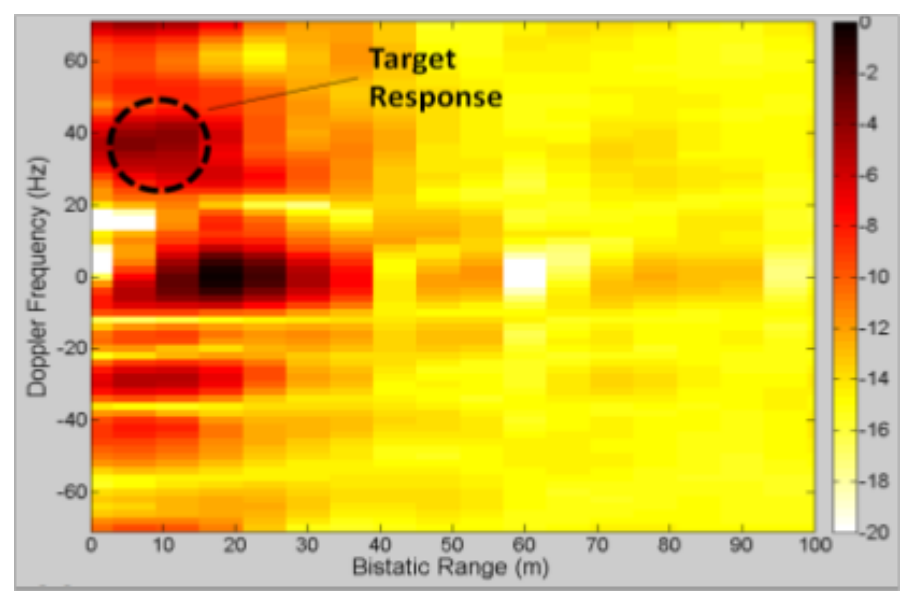

Figure 2.16: Doppler-range plot with target and bistatic range response

(Chetty et al., 2009)

On analyzing figure 2.16 clearly shows that range resolution is less due to the narrow band but multiple object detection accuracy can be improved by using more number of transmitters and the receivers and also with the increase of time integration for data collection. So there is a great possibility of target detection in low clutter and also in high clutter regions using self-ambiguity and the cross ambiguity function analysis.

\section{Concept of using CSI based techniques over RSSI}

Received Signal Strength (RSS), a MAC layer feature, is used for localizing the object using RSS power of the reflected received signal. But for long-range detection of a target, there is a monotonically decrease in received signal strength in which object detection is not accurate. When there is a multipath propagation there is a fluctuation in RSS of $5 \mathrm{db}$ per 600 milliseconds (Zimu Zhou et al.,), using RSS as a parameter does not guarantee the location accuracy. So moving to some robust solution, Channel State Information(CSI) using Channel Impulse Response (CIR), a PHY layer feature, is the best to depict multipath propagation under the time-variant assumption. .

The frequency response of CIR from CSI can be obtained from the present day OFDM based Wireless ( IEEE $802.11 \mathrm{a} / \mathrm{g} / \mathrm{n}$ ) network cards using custom firmware. Each CSI is able to estimate amplitude and phase of each OFDM subcarrier. 


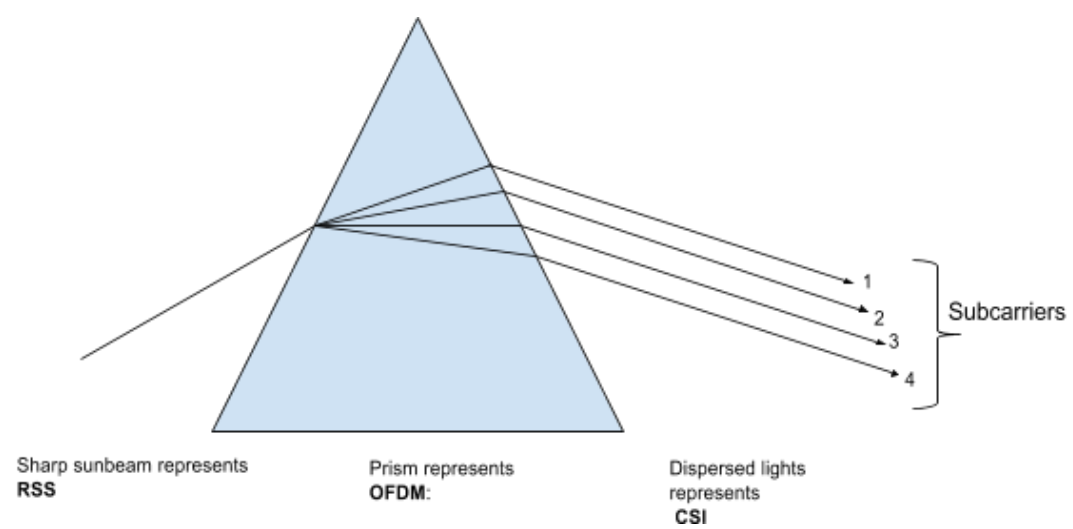

Figure 2.17: Graphical representation of RSS vs CSI

(Zhou et al., 2015)

Illustration of RSS vs CSI in as shown in figure 2.17 represents that RSS is a single valued function (amplitude) superimposed of multipath, but CSI estimates each subcarrier with coarse-grained distinction using CIR in the time domain. Recent studies by many scholars have investigated several methods for amplitude (Zhou et al) and phase (Wu et al) extraction using CSI which can detect the target in LOS and NLOS(using phase orientation).

\subsection{Literature review of the research}

- Guo et al., 2008 - This is the first experiment to detect objects with WiFi signals by using Beacon transmissions such as two parts of DBPSK and DQPSK with DSSS modulation used for transmission for wireless based passive radar. Self-ambiguity function analysis and cross ambiguity analysis is done upon reception of signals for detection.

- Ahamed et al., 2008 - Tracking system for the pervasive environment, linear approximation with RSSI is used by collecting samples by measuring distance and direction of WiFi APs.

- Chetty et al., 2009 - Detection of target using Passive Bistatic WiFi radar when system and target are in high densified echo environment. By using Wifi Access point, reference receiver and surveillance receiver with OFDM, 64 QAM transmission, ambiguity analysis is done for location approximation using bistatic radar concept with the nullification of DSI (Direct Signal Interference). A similar work by the same author (Chetty et al., 2011) is through the wall sensing of personnel using passive bistatic WiFi radar at standoff distances. In this targets are monitored from outside the wall so as to detect the target satisfying the concept of seeing through the walls.

- Falcone et al., 2012 - Tracking of non-stationary objects is done with WiFi-based radar by using Global Positioning System (GPS) for accurate positioning. 
- Rzewuski and Kulpa, 2011 -Designing of system concept for localization using PPDU (PLCP Protocol Data Unit) of MAC address to locate the destination.

- Zhou et al., 2015 - Explained the concept of using Channel State Information (CSI), a PHY feature for extracting CFR and CIR with amplitude and phase detection of the reflected signal. CSI can be obtained from the WiFi network cards with a constraint of using CSI of only 40 $\mathrm{MHz}(802.11 \mathrm{n})$ bandwidth.

- Wu et al., 2015 - Examined the use of CSI for tracking humans, phase, and frequency diversity is used to map body with use of chest motion of a human. The experiment is done for LOS and NLOS detection.

- Van Dijk et al., 2008 - Design of FMCW antenna Array for radar applications with independency in transmitter and receiver reception with Direct Digital Synthesis Technology.

- Adib et al., 2013 - See-Through walls with WiFi, a wall gesture-based communication using inverse sympathetic detection (using only one receiver). Algorithm based detection by calculating the correlation matrix and eigenvalues for separating the signal from the noise along with the indication of direction.

- Adib et al., 2014 - Based on RF Reflections of the body by using static frequency modulated continuous wave (FMCW) where different parts of the body responds for reflecting signals at different frequencies. Here FMCW is operated for some duration, so different body parts reflect at different frequencies and the shape forming of the body is done.

- Adib et al., 2014 - A similar work by using RF reflections for multi personnel localization by using multi-shift FMCW.

- Adib et al., 2015 - Based on variations in reflected signal from the stationary human body monitoring of heartbeat is done. Without loss in generality that these variations are due to heartbeat reflections of a stationary human body at particular reflected frequency of heart with narrow bandwidth.

\subsection{Discussion}

Literature study on several existing methods has shown significant development in designing indoor positioning systems. Some of the methods discussed in 2.8 used RSSI and CSI(Zhou et al., 2015) of the signal for determining characteristics of a signal. Wireless beacon transmission such as DSSS \& DBPSK, DSSS \& DQPSK modulated signal described in Geo et al., 2008 is the first method used for target detection. This shows that beacon transmitted signal changes its characteristics upon reflection from the target and these changed characteristics are used for signal analysis especially with the use of ambiguity function, change in signal characteristics with the transmitted signal is analyzed and this led to determine the characteristics of the target.

Latest research reveals that use of chirp generator described in Adib et al., 2014, Adib et al., 2015 finds its application not only in target detection but also its shape forming. When chip generator 
generates signals with varying frequency in which different body parts of human reflect at different frequencies and these are captured by receiving antennas, upon sample collection and signal processing techniques. This method also improves the accuracy of detection with moderate accuracy in shape forming.

All these systems are capable of detecting target but these methods find difficult in integrating with the existing system because of a closed system and interpretability. However, these methods are the foundation for research in radar engineering, especially in indoor localization.

In the following chapter(s) we discuss some of the basic experimental results done in Telecommunication Laboratory at Asian Institute of Technology, that led to the scope of object detection using narrowband wireless signals is illustrated.

\subsection{Software Validation}

Practical simulation of ambiguity function using (2.11) for DSSS/DBPSK and DSSS/DQPSK using MATLAb is shown in following Figures $2.18,2.19,2.20$ and 2.21
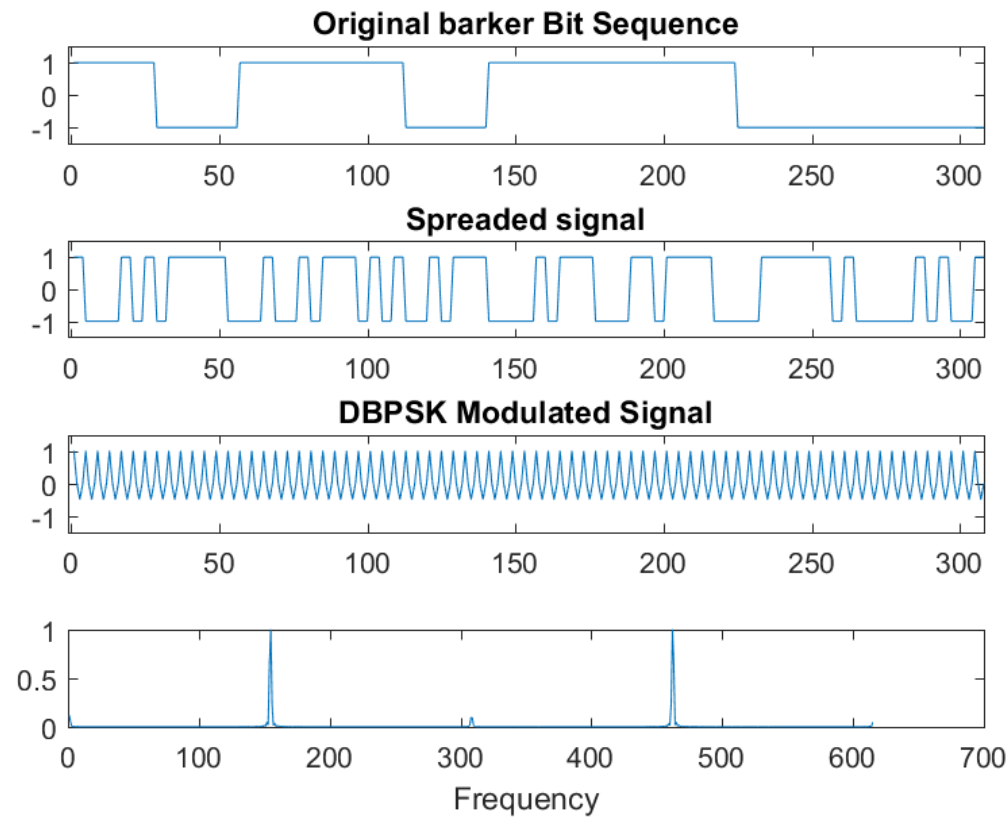

Figure 2.18: Generation of Barker code, Spread signal (DSSS), DBPSK modulated signal and power Spectral density (fft) DSSS/DBPSK signal. 


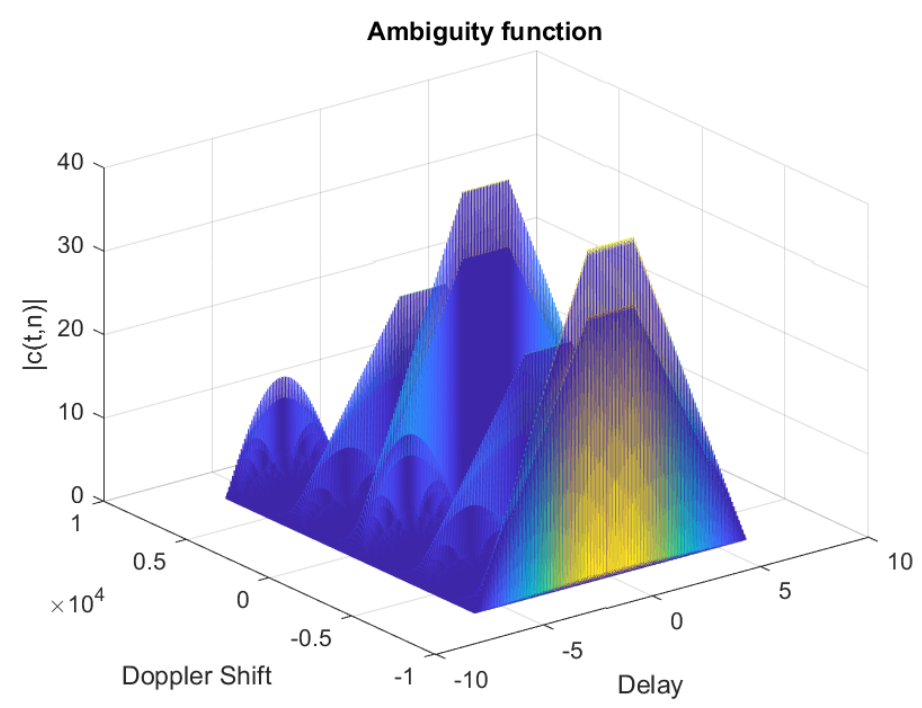

Figure 2.19: Ambiguity function - Doppler shift and delay of DSSS/DBPSK signal

Figure 2.19 represents ambiguity function analysis of a DSSS/DBPSK modulated signal with signal from barker code sequence of bits generated as shown in Figure 2.18. 


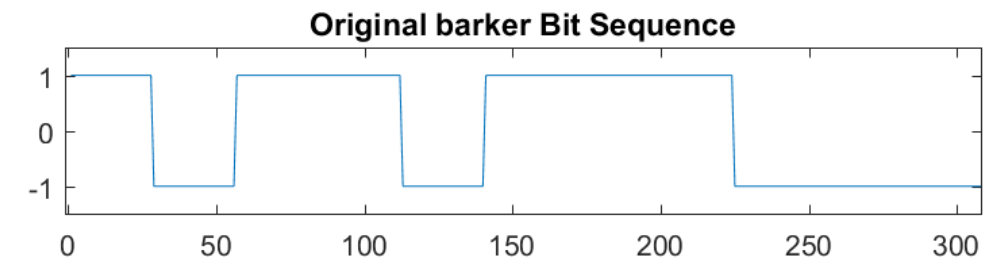

Spreaded signal
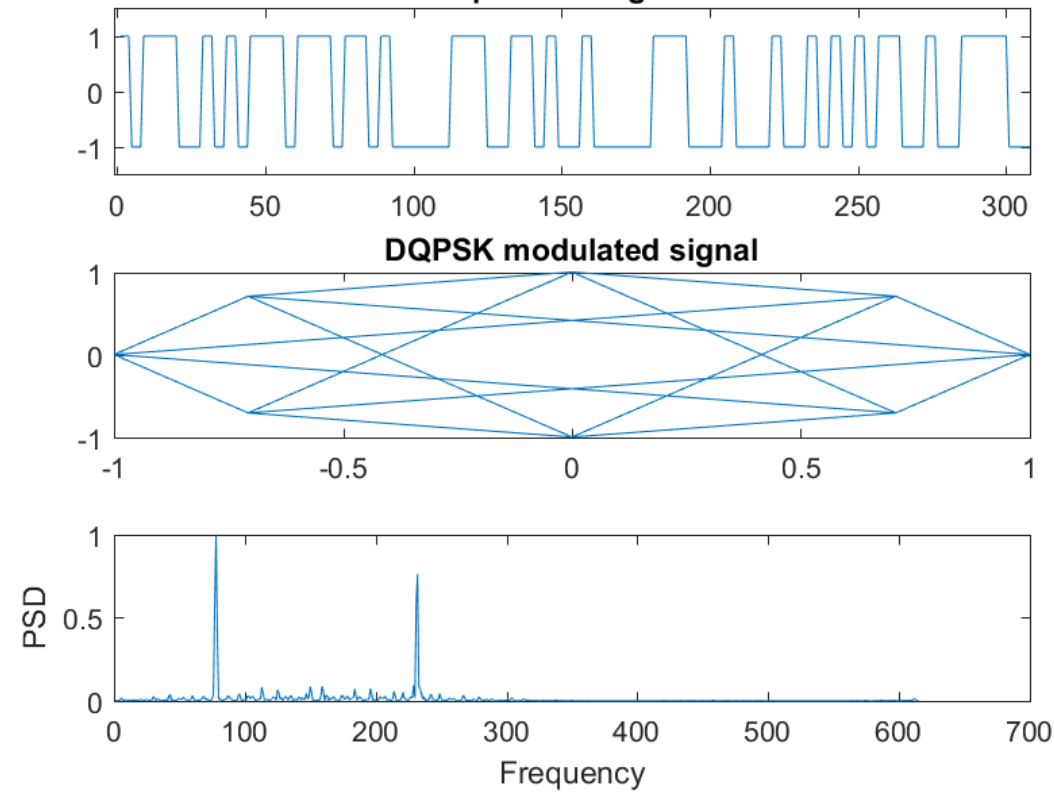

Figure 2.20: Generation of Barher code, spread signal (DSSS), DBPSK modulated signal and power spectral density (fft) DSSS/DQPSK signal. 


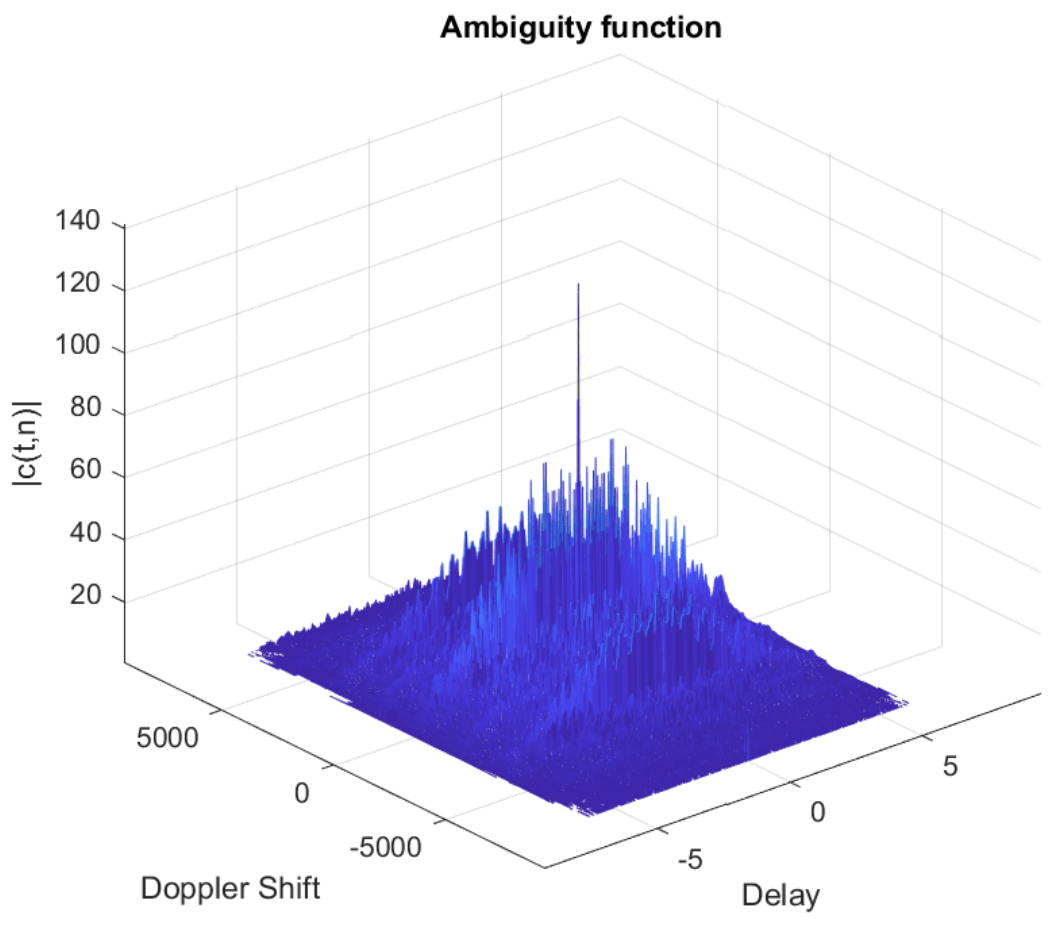

Figure 2.21: Ambiguity function - Doppler shift and dely of DSSS/DQPSK signal

Figure 2.21 represents the ambiguity analysis of a DSSS/DQPSK modulated signal with basic signal from barker sequence of bits as shown in Figure 2.20.

Analysis of ambiguity function from figures 2.19 and 2.21 reveals that peak value is found to be at when there is no Doppler shift and when the delay between the transmitted and the received signal is very less. 


\section{Chapter 3}

\section{Methodology}

\subsection{Power analysis}

At this level, we examine the feasibility of the above proposed model by transmitting and receiving the reflected signal by using available transmitting(Tx) and receiving(Rx) antennas which work in the $\mathrm{MHz}$ up to $\mathrm{GHz}$ frequency ranges. By considering the transmitted signal power level and received signal power level and by implementing digital signal processing techniques we can obtain the distance at which the object is located. For this, we need to do the following.

1. Tx Antenna : A transmitting antenna which is readily available for experimentation and which generates from $\mathrm{MHz}$ to $\mathrm{GHz}$ frequencies is needed for physical experimentation.

2. Rx Antenna : An antenna capable of detecting the signal having frequencies from $\mathrm{MHz}$ to $\mathrm{GHz}$ frequencies.

3. Signal generator which is connected to Tx antenna and Oscilloscope connected to Rx antenna as shown in figure 3.1 is used to primarily transmit and receive the signal.

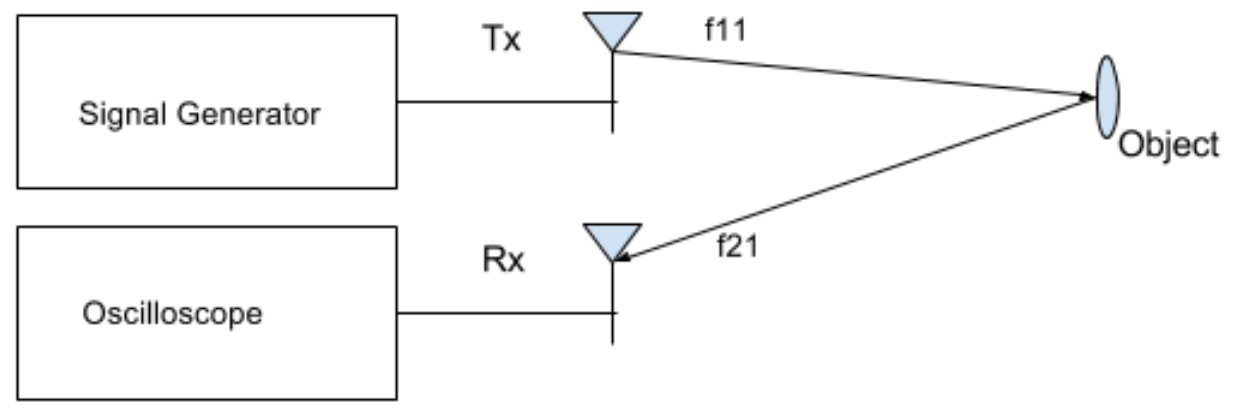

Figure 3.1: Basic model using signal generator and spectrum analyser for feasibility test (Tx and $\mathrm{Rx}$ antennas of $2.4 \mathrm{GHz}$ are available in AIT TC Lab) 


\section{Experimentation}

Basic experimentation is done in Telecommunication laboratory of Asian Institute of Technology to demonstrate the scope of object detection using wireless signals of narrowband frequency. Experimentation procedure follows the later stages of the chapter.

For object detection, primary experiment using Signal generator $(0-3 \mathrm{GHz})$, Spectrum analyzer $(0$ $3 \mathrm{GHz}$ ), one transmitting(Tx) antenna and one receiving $(\mathrm{Rx})$ is performed.

1. Transmitting (Tx) antenna is attached to the RF output of the signal generator and receiving $(\mathrm{Rx})$ is attached to the RF input of the Spectrum analyzer.

2. Antennas are kept at a nominal distance of $3 \mathrm{~cm}$ as shown in figure 3.2 to test the signal transmission and receiving capabilities. RF signal is transmitted with $-10 \mathrm{dBm}$ amplitude and varying frequency as shown in table 3.1 to see at which frequencies that the antennas have higher gain. Two values of the received signal are recorded. Generally, Tx and Rx antennas are designed to work optimal at $2.2 \mathrm{GHz}$ to $2.6 \mathrm{GHz}$ with center frequency $2.4 \mathrm{GHz}$. 


\begin{tabular}{|l|l|l|l|}
\hline Tx Frequency in & $\begin{array}{l}\text { Tx amplitude in } \\
\text { GHz }\end{array}$ & $\begin{array}{l}\text { Rx Frequency in GHz } \\
\text { at peak amp }\end{array}$ & $\begin{array}{l}\text { Rx amplitude in } \\
\mathbf{d B m}\end{array}$ \\
\hline 1.5 & -10 & 1.5013 & -44.9 \\
\hline & & & -45 \\
\hline 1.75 & -10 & 1.7508 & -50 \\
\hline & & & -49 \\
\hline 2 & -10 & 2.003 & -44.5 \\
\hline & & & -43 \\
\hline 2.2 & -10 & 2.198 & -41.23 \\
\hline & & & -41.58 \\
\hline 2.4 & -10 & 2.40004 & -38.241 \\
\hline & & & -37.6 \\
\hline 2.6 & -10 & 2.5981 & -38.45 \\
\hline & & & -38.61 \\
\hline 2.8 & -10 & -2.8005 & -46 \\
\hline & & & -47.74 \\
\hline 3 & -10 & 2.9982 & -49.62 \\
\hline & & & -50.48 \\
\hline
\end{tabular}

Table 3.1: Rx and Tx values at $-10 \mathrm{dBm}$ Tx amplitude with varying frequency with $3 \mathrm{~cm}$ distance apart betwenw Tx and Rx when no obstacle is introduced 


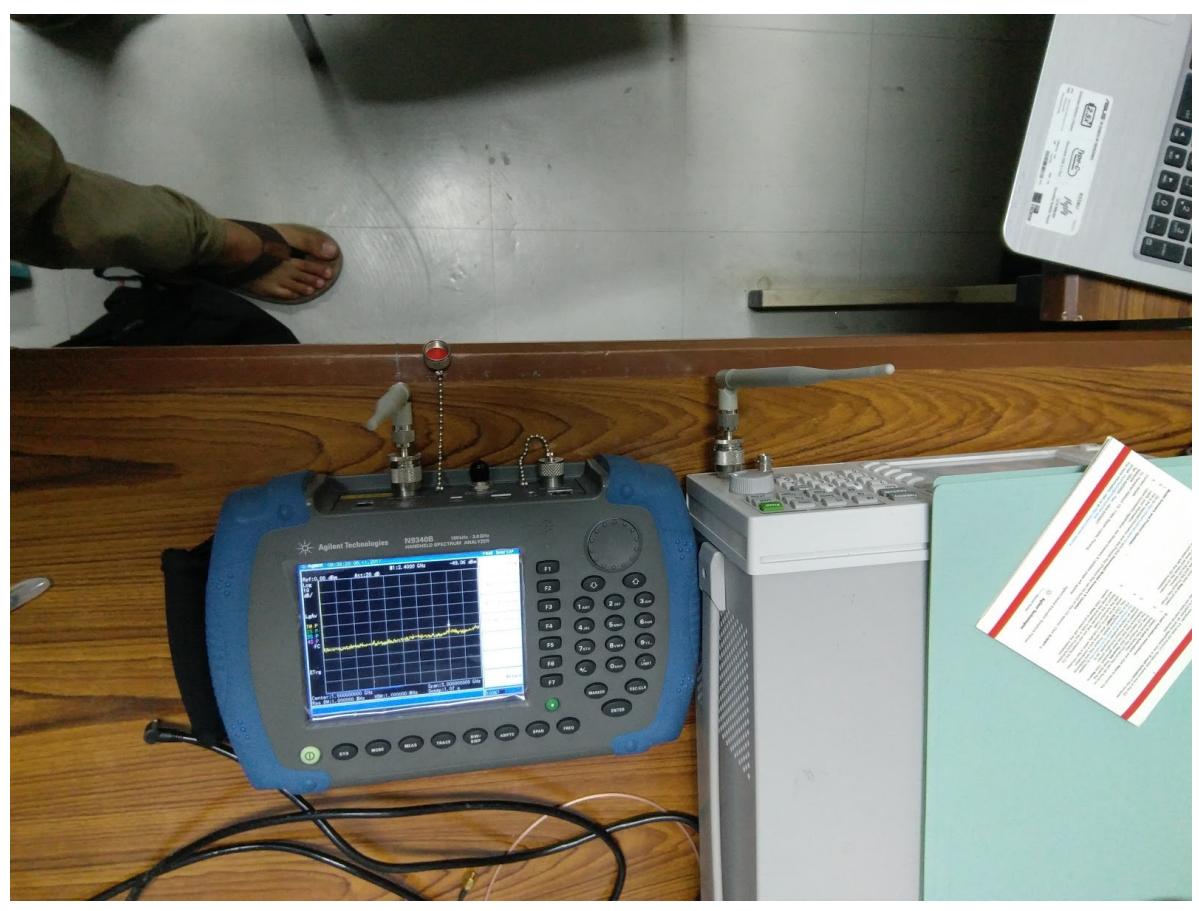

Figure 3.2: when Tx-Rx are at $3 \mathrm{~cm}$ apart and when no obstacle is introduced

3. Tx and Rx are placed at $30 \mathrm{~cm}$ apart and the RF signal is generated with $2.4 \mathrm{GHz},-10 \mathrm{dBm}$ amplitude. Spectrum analyser clearly shows peak values (are recorded as shown in Tables 3.2, 3.3 , and 3.4 when experiment is repeated for 3 times) at $2.4 \mathrm{Ghz}$ where other values are noise signals with negligible amplitude as shown in the Figure 3.3

\begin{tabular}{|c|c|c|c|c|}
\hline $\begin{array}{l}\text { Tx Frequency } \\
\text { in GHz }\end{array}$ & $\begin{array}{l}\text { Tx amplitude in } \\
\mathrm{dBm}\end{array}$ & $\begin{array}{l}\text { Rx Frequency in } \\
\text { GHz at peak amp }\end{array}$ & $\begin{array}{l}\text { Rx amplitude } \\
\text { in } \mathbf{d B m}\end{array}$ & $\begin{array}{l}\text { Average Rx } \\
\text { amplitude in } \\
\text { dBm }\end{array}$ \\
\hline 2.4 & -10 & 2.40 & -41.2 & \\
\hline 2.4 & -10 & 2.40 & -41.84 & \\
\hline 2.4 & -10 & 2.40 & -41.43 & \\
\hline 2.4 & -10 & 2.40 & -41.21 & \\
\hline 2.4 & -10 & 2.40 & -41.26 & \\
\hline 2.4 & -10 & 2.40 & -41.15 & -41.4414 \\
\hline
\end{tabular}

Table 3.2: Receiver (Rx) observations recorded first (1st) time with distance between Tx and $\mathrm{Rx}$ as $30 \mathrm{~cm}$, no obstacle introduced 


\begin{tabular}{|l|l|l|l|l|}
\hline Tx Frequency & Tx amplitude in & Rx Frequency in & Rx amplitude \\
in GHz & dBm & GHz at peak amp & in dBm & $\begin{array}{l}\text { amplitude in } \\
\text { dBm }\end{array}$ \\
\hline 2.4 & -10 & 2.40 & -43.70 & \\
\hline 2.4 & -10 & 2.40 & -43.30 & \\
\hline 2.4 & -10 & 2.40 & -43.07 & \\
\hline 2.4 & -10 & 2.40 & -43.92 & \\
\hline 2.4 & -10 & 2.40 & -43.27 & \\
\hline 2.4 & -10 & 2.40 & -44.10 & \\
\hline 2.4 & -10 & 2.40 & -43.60 & \\
\hline 2.4 & -10 & 2.40 & -42.83 & \\
\hline 2.4 & -10 & 2.40 & -43.76 & \\
\hline 2.4 & -10 & 2.40 & -44.15 & \\
\hline 2.4 & -10 & 2.40 & -689 \\
\hline
\end{tabular}

Table 3.3: Receiver (Rx) observations recorded second (2nd) time with distance between $\mathrm{Tx}$ and $\mathrm{Rx}$ as $30 \mathrm{~cm}$, no obstacle introduced 


\begin{tabular}{|l|l|l|l|l|}
\hline Tx Frequency & Tx amplitude in & Rx Frequency in & Rx amplitude & $\begin{array}{l}\text { Average Rx } \\
\text { amplitude in } \\
\text { in GHz }\end{array}$ \\
\hline 2.4 & -10 & 2.40 & -44.59 & dBm dBm \\
\hline 2.4 & -10 & 2.40 & -43.57 & \\
\hline 2.4 & -10 & 2.40 & -44.99 & \\
\hline 2.4 & -10 & 2.40 & -43.64 & \\
\hline 2.4 & -10 & 2.40 & -44.34 & \\
\hline 2.4 & -10 & 2.40 & -45.04 & \\
\hline 2.4 & -10 & 2.40 & -45.21 & \\
\hline 2.4 & -10 & 2.40 & -43.61 & -44.292 \\
\hline 2.4 & -10 & 2.40 & -44.05 & \\
\hline 2.4 & -10 & 2.40 & -43.89 & \\
\hline
\end{tabular}

Table 3.4: Receiver ( $\mathrm{Rx}$ ) observations recorded third (3rd) time with distance between $\mathrm{Tx}$ and $\mathrm{Rx}$ as $30 \mathrm{~cm}$, no obstacle introduced 


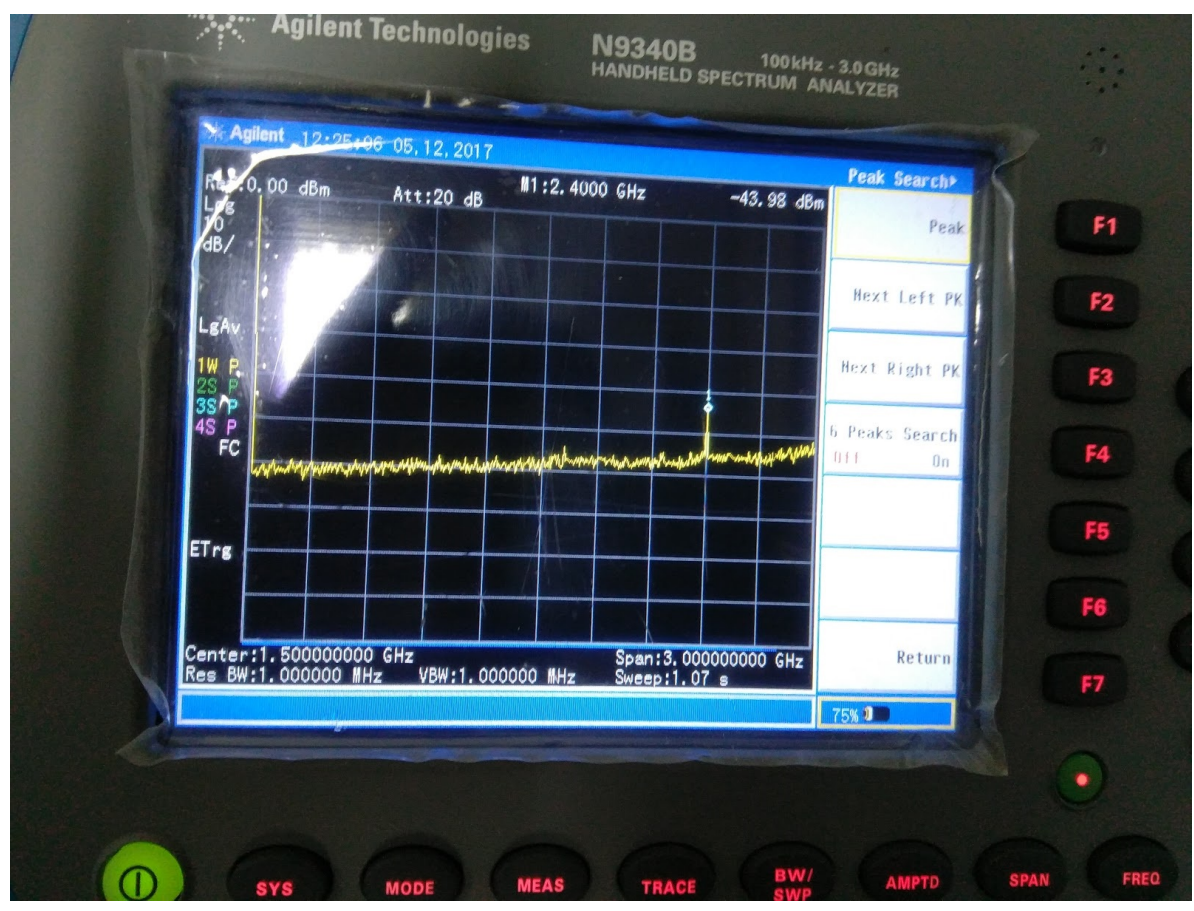

Figure 3.3: Received Peak value observation when Tx signal frequency is $2.4 \mathrm{GHz},-10 \mathrm{dBm}$

4. Experimentation scenario is shown in figure 3.4. Now the obstacle is introduced at different distance (LOS) ranging from left of transmitter(Tx) to the receivers(Rx) right scaling from $15 \mathrm{~cm}$ to $45 \mathrm{~cm}$ as shown in Figure 3.4 and observations are recorded in Tables 3.5, 3.6 and 3.7 (when experiment is repeated for 3 times) with a stamp for every $3 \mathrm{~cm}$.

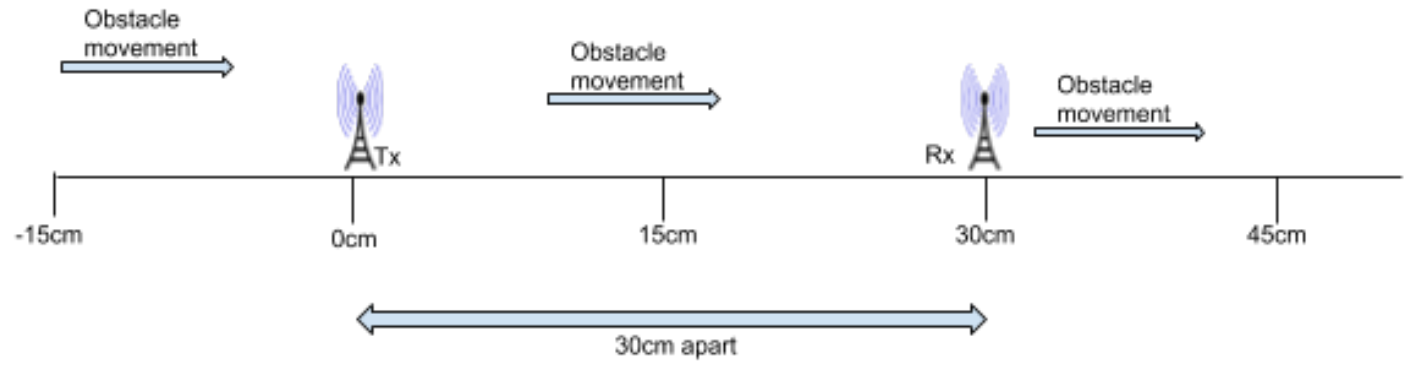

Figure 3.4: Experimentation scenario 


\begin{tabular}{|c|c|c|c|c|c|c|c|}
\hline $\begin{array}{l}\text { Obstacle } \\
\text { distance. } \\
\text { From } \mathbf{T x} \\
\text { to } \mathbf{R x}\end{array}$ & $\begin{array}{l}\text { Rx } \\
\text { amp } \\
\text { dBm1 }\end{array}$ & $\begin{array}{l}\text { Rx } \\
\text { amp } \\
\text { dBm2 }\end{array}$ & $\begin{array}{l}\text { Rx } \\
\text { amp } \\
\text { dBm3 }\end{array}$ & $\begin{array}{l}\text { Rx } \\
\text { amp } \\
\text { dBm4 }\end{array}$ & $\begin{array}{l}\text { Rx } \\
\text { amp } \\
\text { dBm5 }\end{array}$ & $\begin{array}{l}\text { Rx } \\
\text { amp } \\
\text { dBm6 }\end{array}$ & $\begin{array}{l}\text { Rx amp } \\
\text { dBm Avg }\end{array}$ \\
\hline-15 & -43.17 & -43.2 & -43.57 & -43.77 & -43.13 & -43.29 & -43.355 \\
\hline-12 & -43.67 & -42.9 & -43.15 & -43.56 & -43.14 & -42.97 & -43.231 \\
\hline-9 & -42.56 & -42.57 & -43.15 & -42.03 & -42.18 & -42.33 & -42.47 \\
\hline-6 & -44.8 & -45.61 & -45.36 & -44.34 & -44.72 & -45.12 & -44.9916 \\
\hline-3 & -41.75 & -41.88 & -43.21 & -43.35 & -43.1 & -42.95 & -42.706 \\
\hline 0 & -52.8 & -51.42 & -53.7 & -54.08 & -54.42 & -55.6 & -53.67 \\
\hline 3 & -46.71 & -47.01 & -47.64 & -47.5 & -47.05 & -46.63 & -47.09 \\
\hline 6 & -45.25 & -44.64 & -46.64 & -46.29 & -47.07 & -46.23 & -46.02 \\
\hline 9 & -46.32 & -45.05 & -46.11 & -46.48 & -46.5 & -47.73 & -46.365 \\
\hline 12 & -49.83 & -46.97 & -46.79 & -47.02 & -47.3 & -46.14 & -47.3416 \\
\hline 15 & -44.39 & -44.63 & -44.68 & -44.24 & -45.08 & -44.61 & -44.605 \\
\hline 18 & -46.84 & -46.84 & -46.25 & -46.49 & -46.1 & -47.14 & -44.61 \\
\hline 21 & -43.3 & -43.34 & -43.73 & -43.57 & -44.1 & -43.07 & -43.5183 \\
\hline 24 & -42.75 & -43.96 & -44.91 & -44.31 & -44.9 & -44.95 & -44.2966 \\
\hline 27 & -48.69 & -48.12 & -49.91 & -47.79 & -48.39 & -47.31 & -48.368 \\
\hline 30 & -51.31 & -49.67 & -52.17 & -50.86 & -52.56 & -52.02 & -51.4316 \\
\hline 33 & -42.41 & -41.81 & -42.38 & -42.5 & -41.44 & -42.15 & -42.511 \\
\hline 36 & -43.27 & -43.2 & -43.28 & -43.31 & -43.53 & -43.51 & -43.35 \\
\hline 39 & -44.58 & -45.96 & -45.7 & -46.21 & -45.79 & -46.37 & -45.7688 \\
\hline 42 & -43.56 & -43.39 & -43.43 & -43.81 & -43.26 & -43.4 & -43.475 \\
\hline 45 & -43.14 & -42.79 & -43.07 & -43.67 & -42.74 & -43.14 & -43.091 \\
\hline
\end{tabular}

Table 3.5: Receiver $(\mathrm{Rx}) \mathrm{dBm}$ recorded first (1st) time at various distance of obstacle position from the Tx 


\begin{tabular}{|c|c|c|c|c|c|c|c|}
\hline $\begin{array}{l}\text { Obstacle } \\
\text { distance. } \\
\text { From Tx } \\
\text { to } \mathbf{R x}\end{array}$ & $\begin{array}{l}\text { Rx } \\
\text { amp } \\
\text { dBm1 }\end{array}$ & $\begin{array}{l}\text { Rx } \\
\text { amp } \\
\text { dBm2 }\end{array}$ & $\begin{array}{l}\text { Rx } \\
\text { amp } \\
\text { dBm3 }\end{array}$ & $\begin{array}{l}\text { Rx } \\
\text { amp } \\
\text { dBm4 }\end{array}$ & $\begin{array}{l}\text { Rx } \\
\text { amp } \\
\text { dBm5 }\end{array}$ & $\begin{array}{l}\text { Rx } \\
\text { amp } \\
\text { dBm6 }\end{array}$ & $\begin{array}{l}\text { Rx amp } \\
\text { dBm Avg }\end{array}$ \\
\hline-15 & -45.79 & -44.47 & -44.73 & -46.57 & -45.33 & -44.54 & -45.22666 \\
\hline-12 & -45.3 & -44.9 & -44.58 & -44.07 & -44.54 & -45.33 & -44.7869 \\
\hline-9 & -46.6 & -44.63 & -47.18 & -46.5 & -45.52 & -46.21 & -46.1066 \\
\hline-6 & -45.16 & -45.13 & -45.23 & -44.18 & -45.03 & -44.83 & -44.9266 \\
\hline-3 & -45.21 & -45.2 & -46.13 & -47.2 & -45 & -45.23 & -45.6616 \\
\hline 0 & -55.9 & -55.25 & -54.63 & -55 & -54.68 & -55.37 & -55.1383 \\
\hline 3 & -51 & -50.29 & -50.87 & -50.76 & -50.72 & -50.81 & -50.74166 \\
\hline 6 & -49.21 & -50 & -48.79 & -50.06 & -50.13 & -50.15 & -49.7233 \\
\hline 9 & -48.16 & -46.7 & -47.06 & -49.13 & -47.02 & -47.6 & -47.6116 \\
\hline 12 & -50.74 & -51.17 & -48.18 & -49.47 & -50.09 & -50.46 & -50.01833 \\
\hline 15 & -47.22 & -48.69 & -47.9 & -48.4 & -47.79 & -48.42 & -48.07 \\
\hline 18 & -48.83 & -50 & -49.79 & -50.69 & -51.55 & -50.51 & -50.2833 \\
\hline 21 & -48.69 & -47.33 & -49.63 & -50.17 & -50.7 & -49.52 & -49.3 \\
\hline 24 & -50.18 & -51.45 & -52.83 & -53.58 & -51.21 & -51.98 & -51.87 \\
\hline 27 & -53.8 & -52.85 & -52.22 & -51.41 & -54 & -53.8 & -53.0133 \\
\hline 30 & -55.58 & -55.68 & -54.73 & -55.52 & -55.29 & -55.35 & -55.3583 \\
\hline 33 & -46.7 & -47.13 & -48.29 & -46.31 & -47.61 & -48.3 & -47.39 \\
\hline 36 & -44.9 & -44.21 & -45.17 & -45.13 & -45.09 & -45.3 & -44.9666 \\
\hline 39 & -46.78 & -46.57 & -46.51 & -46.15 & -46.16 & -46.17 & -46.39 \\
\hline 42 & -44.6 & -44.36 & -44.74 & -44.74 & -45.11 & -44.1 & -44.6083 \\
\hline 45 & -46.11 & -45.9 & -45.32 & -45.29 & -45.77 & -45.38 & -45.6833 \\
\hline
\end{tabular}

Table 3.6: Receiver $(\mathrm{Rx}) \mathrm{dBm}$ recorded second (2nd) time at various distance of obstacle position from the $\mathrm{Tx}$ 


\begin{tabular}{|c|c|c|c|c|c|c|c|}
\hline $\begin{array}{l}\text { Obstacle } \\
\text { distance. } \\
\text { From Tx } \\
\text { to } \mathbf{R x}\end{array}$ & $\begin{array}{l}\text { Rx } \\
\text { amp } \\
\text { dBm1 }\end{array}$ & $\begin{array}{l}\text { Rx } \\
\text { amp } \\
\text { dBm2 }\end{array}$ & $\begin{array}{l}\text { Rx } \\
\text { amp } \\
\text { dBm3 }\end{array}$ & $\begin{array}{l}\text { Rx } \\
\text { amp } \\
\text { dBm4 }\end{array}$ & $\begin{array}{l}\text { Rx } \\
\text { amp } \\
\text { dBm5 }\end{array}$ & $\begin{array}{l}\text { Rx } \\
\text { amp } \\
\text { dBm6 }\end{array}$ & $\begin{array}{l}\text { Rx amp } \\
\text { dBm Avg }\end{array}$ \\
\hline-15 & -45.09 & -45.41 & -45.46 & -45.42 & -45.69 & -45.4 & -45.4116 \\
\hline-12 & -43.05 & -43.21 & -43.76 & -42.73 & -42.86 & -43.21 & -43.3166 \\
\hline-9 & -45.1 & -44.79 & -45.13 & -44.12 & -44.57 & -44.52 & -44.705 \\
\hline-6 & -44.4 & -43.52 & -42.09 & -43.51 & -43.55 & -43.44 & -43.41833 \\
\hline-3 & -46.52 & -47.88 & -47.74 & -46.24 & -45.96 & -47.61 & -46.9916 \\
\hline 0 & -50.56 & -51.13 & -52.71 & -50.35 & -49.35 & -52.61 & -51.1183 \\
\hline 3 & -47.43 & -47.03 & -47.08 & -47.98 & -47.49 & -46.5 & -47.2561 \\
\hline 6 & -48.54 & -49.72 & -49.5 & -49.13 & -50.46 & -49.35 & -49.45 \\
\hline 9 & -45.75 & -46.92 & -47.03 & -45.95 & -46.2 & -46.7 & -46.425 \\
\hline 12 & -46.99 & -47.33 & -48.88 & -47.54 & -48.57 & -47.16 & -47.745 \\
\hline 15 & -45.81 & -46.92 & -47.03 & -45.95 & -46.2 & -46.7 & -46.435 \\
\hline 18 & -45.73 & -47.17 & -46 & -46.97 & -46.44 & -46.62 & -46.4883 \\
\hline 21 & -46.99 & -47.07 & -47 & -46.62 & -46.67 & -46.61 & -46.8266 \\
\hline 24 & -46.88 & -47.59 & -48.4 & -48.21 & -47.29 & -47.18 & -47.5916 \\
\hline 27 & -49 & -48.88 & -49.94 & -49.1 & -49.49 & -48.9 & -49.21833 \\
\hline 30 & -51.84 & -51.16 & -49.55 & -50.21 & -49.71 & -50.25 & -50.4533 \\
\hline 33 & -46.7 & -47.13 & -48.29 & -46.31 & -47.61 & -48.3 & -47.39 \\
\hline 36 & -44.9 & -44.21 & -45.17 & -45.13 & -45.3 & -45.09 & -44.9666 \\
\hline 39 & -46.79 & -46.57 & -46.51 & -46.51 & -46.15 & -46.16 & -46.44833 \\
\hline 42 & -44.65 & -44.36 & -44.74 & -44.74 & -45.11 & -44.1 & -44.61666 \\
\hline 45 & -46.11 & -45.9 & -45.32 & -45.29 & -45.77 & -45.58 & -45.66166 \\
\hline
\end{tabular}

Table 3.7: Receiver ( $\mathrm{Rx}) \mathrm{dBm}$ recorded third (3rd) time at various distance of obstacle position from the Tx 
5. At each value of distance for every $3 \mathrm{~cm}$ of obstacle from the $\mathrm{Tx}$, Six readings are recorded and average $\mathrm{Rx}$ amplitude $\mathrm{dBm}$ is calculated as Rx amplitude $\mathrm{dBm}$ average for every position. Graphs as shown in figures 3.5, 3.6 and 3.7 (when experiment is repeated for 3 times) is plotted for Rx amp dBm avg v/s distance and analysis of graph is done before and after introducing obstacle.

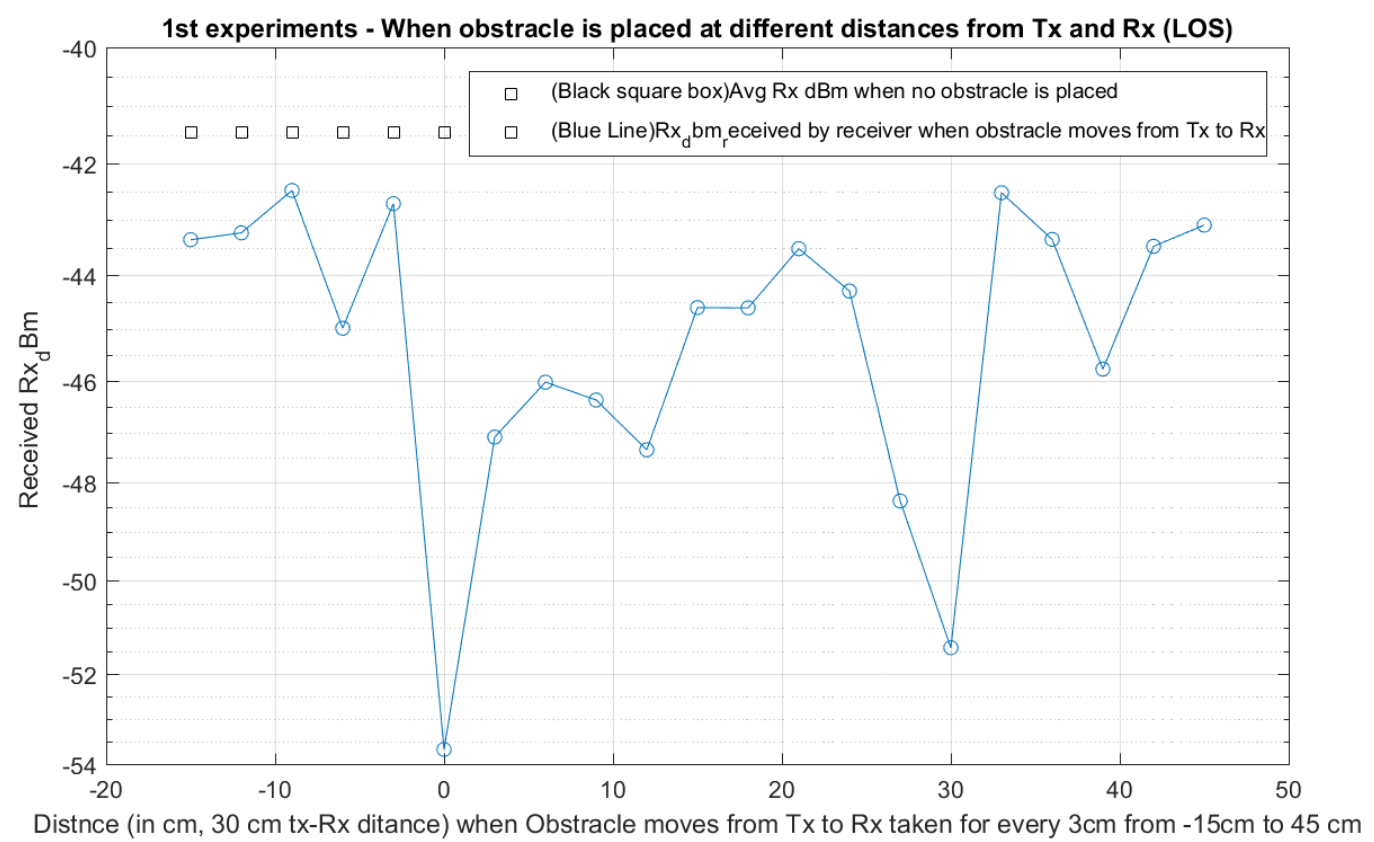

Figure 3.5: Experiment 1: Graph comparing $\mathrm{Rx}$ amp dBm avg when obstacle is at different distances(LOS) b/w Tx and Rx, plotted for every $3 \mathrm{~cm}$ of move 


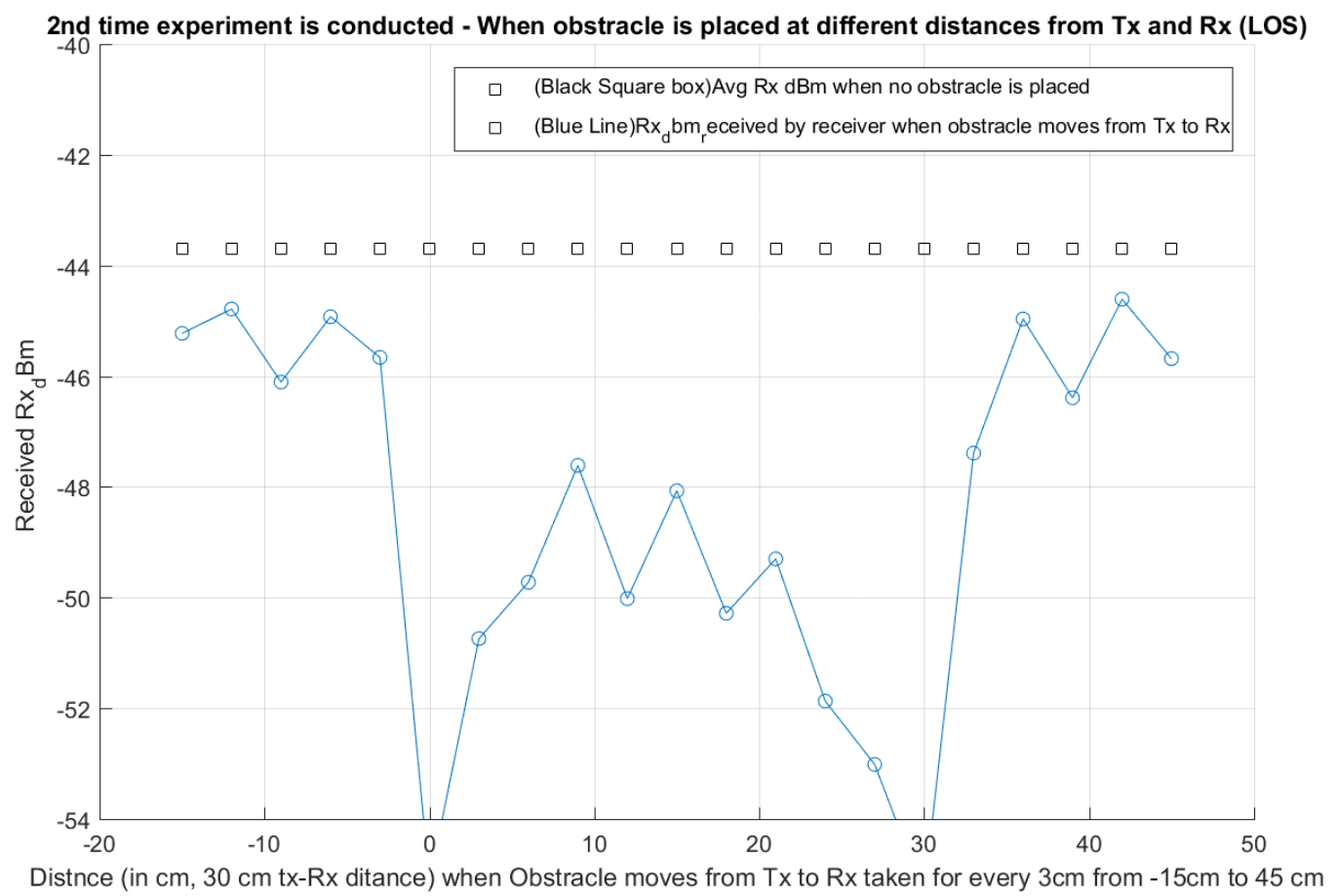

Figure 3.6: Experiment 2: Graph comparing $\mathrm{Rx}$ amp $\mathrm{dBm}$ avg when obstacle is at different distances(LOS) b/w Tx and Rx, plotted for every $3 \mathrm{~cm}$ of move

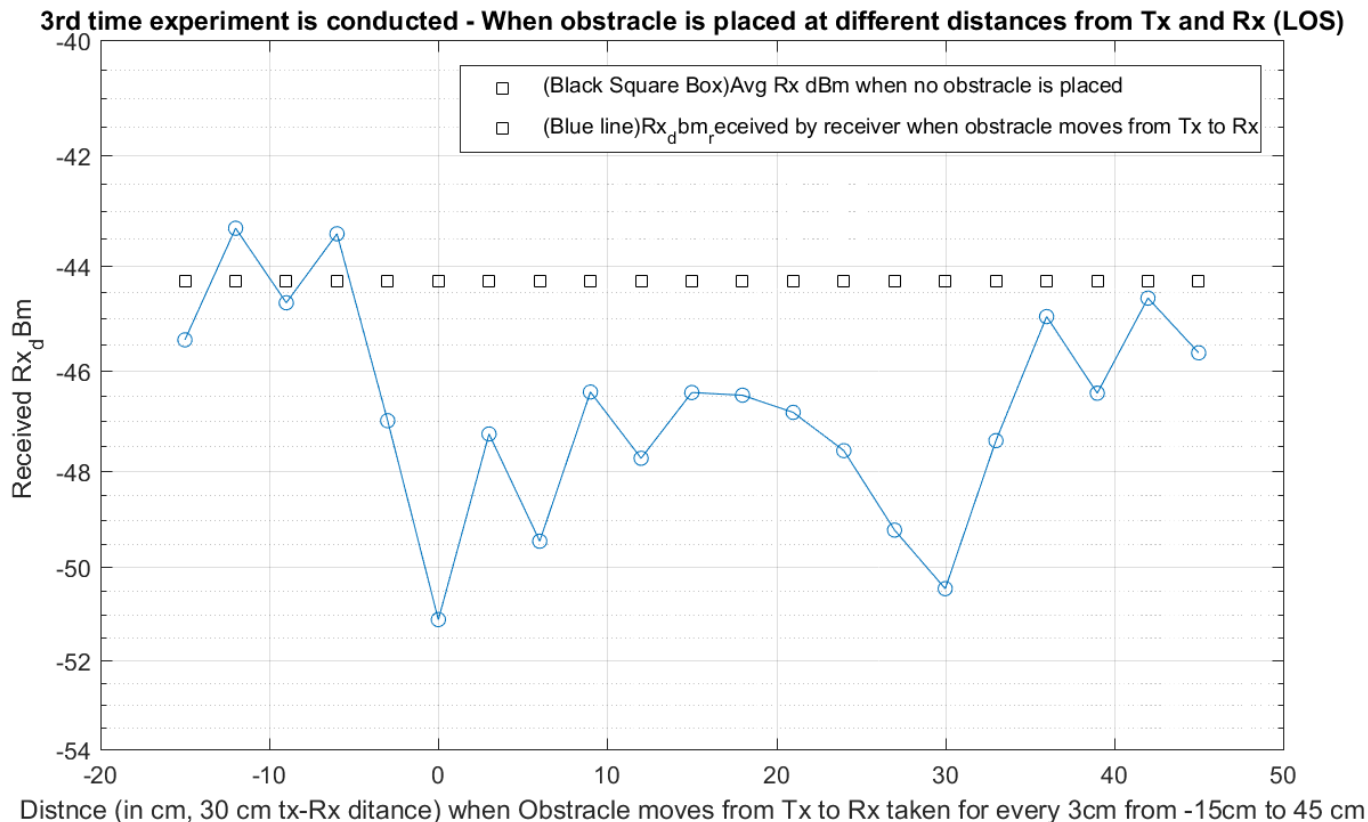

Figure 3.7: Experiment 3: Graph comparing $\mathrm{Rx}$ amp $\mathrm{dBm}$ avg when obstacle is at different distances(LOS) b/w Tx and Rx, plotted for every $3 \mathrm{~cm}$ of move 


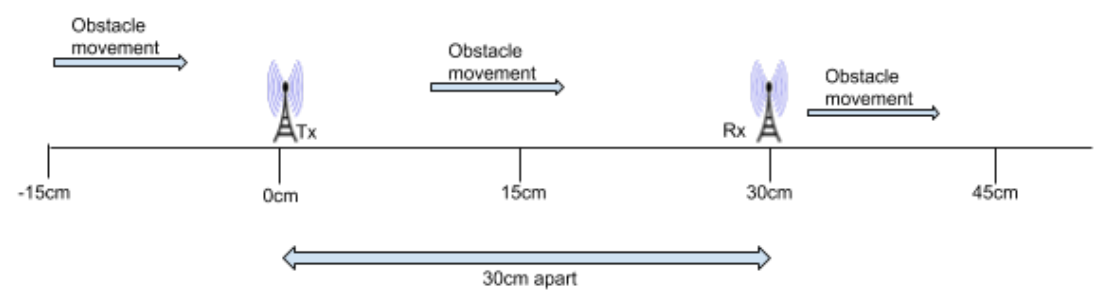

Figure 3.8: Experimentation Scenario

\section{Graph Analysis}

Figures $3.5,3.6$ and 3.7 is a graphical signal representation of experimentation scenario in Figure 3.8. For analysis we divide the scenario into four parts such as left of $T x(-15 \mathrm{~cm}$ to $0 \mathrm{~cm})$, right of $\operatorname{Tx}(0 \mathrm{~cm}$ to $15 \mathrm{~cm})$, left of $\operatorname{Rx}(15 \mathrm{~cm}$ to $30 \mathrm{~cm})$ and right of $(30 \mathrm{~cm}$ to $45 \mathrm{~cm})$.

1. Right of $\mathbf{T x}(0 \mathrm{~cm}$ to $15 \mathrm{~cm})$ : when obstacle is introduced and moved from $0 \mathrm{~cm}$ to $15 \mathrm{~cm}$ we could see that $\mathrm{Rx} \mathrm{dBm}$ increases till the mid value of right of $\mathrm{Tx}(\mathrm{ocm}$ to $15 \mathrm{~cm})$, about $7.5 \mathrm{~cm}$ then the graph starts decreases from that midpoint to endpoint of Right of $\operatorname{Tx}(0 \mathrm{~cm}$ to $15 \mathrm{~cm})$, i.e mid point of Tx-Rx distance. Here the effect of small sidelobes are reduced till the quarter distance of $30 \mathrm{~cm}$ due to which the graph increases and in the next quarter often sidelobes effect is not nullified and the signal reflection from obstacle can cause a decrease of signal level due to which graph falls down.

2. Left of $\operatorname{Rx}(15 \mathrm{~cm}$ to $30 \mathrm{~cm})$ : from mid point of Tx-Rx, graph starts increasing till the mid point of Left of $\operatorname{Rx}(15 \mathrm{~cm}$ to $30 \mathrm{~cm})$, about at $21 \mathrm{~cm}$ and then the graphs start decreasing until the end point of Left of $\operatorname{Rx}(15 \mathrm{~cm}$ to $30 \mathrm{~cm})$, i.e at $30 \mathrm{~cm}$ near the receiver. The value of received $\mathrm{dBm}$ is low at $30 \mathrm{~cm}$ point because of blockage of the signal from the transmitter(Tx) to the receiver(Rx) by the obstacle.

3. Left of $\mathbf{T x}(\mathbf{- 1 5} \mathbf{c m}$ to $\mathbf{0 ~} \mathbf{~ c m})$ : Similar effect, Transmitted 1 power level is less when obstacle is at $\operatorname{Tx}(0 \mathrm{~cm})$ and it increases as the obstacle moves from farther left of Tx and starts decreasing from the quarter point of $(0 \mathrm{~cm}$ to $-15 \mathrm{~cm})$ i.e the effect of side lobe is observed and it starts increasing from point the mid point when the sidelobe effect is reduced. And then the reflections from the main lobe due to the obstacle can cause the signal to interference and the signal attenuation is observed at the receiver.

4. Right of $\operatorname{Rx}(30 \mathrm{~cm}$ to $45 \mathrm{~cm})$ : Similar effect of sidelobe and main lobe reflection by the obstacle when it moves from right to farther right of Rx can cause increase and decrease of signal power. 


\section{Environmental conditions :}

1. High clutter region having many computers in the lab.

2. Mobile phone signal interface.

3. Two air conditioners are switched on.

4. Several dielectric materials such as antenna under power off condition.

\section{Conclusions on the power analysis}

From the graphs figures 3.5, 3.6 and 3.7 are clearly symmetrical and clearly shows that the object detection parameters almost same when experiment is repeated for 3 times.

The ratio of Average received signal $\mathrm{dBm}$ when no object to the Received signal $\mathrm{dBm}$ when object is introduced at instantaneous distance from Tx is approximately equal for all the 3 experiments.

$$
\begin{aligned}
& \text { Experiment } 1\left(\frac{A v g R x d B m \text { received when no obstacle }}{R x d B m \text { received instantaneously when obstacle is present }}\right) \\
& \text { Experiment } 2\left(\frac{A v g R x d B m \text { received when no obstacle }}{R x d B m \text { received instantaneously when obstacle is present }}\right) \\
& \text { Experiment } 3\left(\frac{A v g R x d B m \text { received when no obstacle }}{R x d B m \text { received instantaneously when obstacle is present }}\right)
\end{aligned}
$$

From this we can write that, $3.1 \approx 3.2 \approx 3.3$. 


\subsection{Time delay analysis}

\section{Object detection using FMCW signal}

Simulation is performed using GNU Radio, a Software Defined Radio,for detecting object using FMCW signal.

The simulation includes generation of FMCW signal using sawtooth waveform with Voltage Controlled Waveform (Carrier signal). A signal is transmitted and delay is introduced after receiving the signal before processing the signal with the transmitted signal.

\begin{tabular}{|l|l|}
\hline Chirp signal & Sawtooth \\
Chirp Frequency & $1 \mathrm{kHz}$ \\
Band width of FMCW & $750 \mathrm{kHz}$ \\
Sampling rate & $3 \mathrm{MSPS}$ \\
\hline
\end{tabular}

Table 3.8: Simulation Parameters

Processing of signals are shown in figure 3.9 and Simulation parameters are implemented in GNU radio as shown in figure 3.10

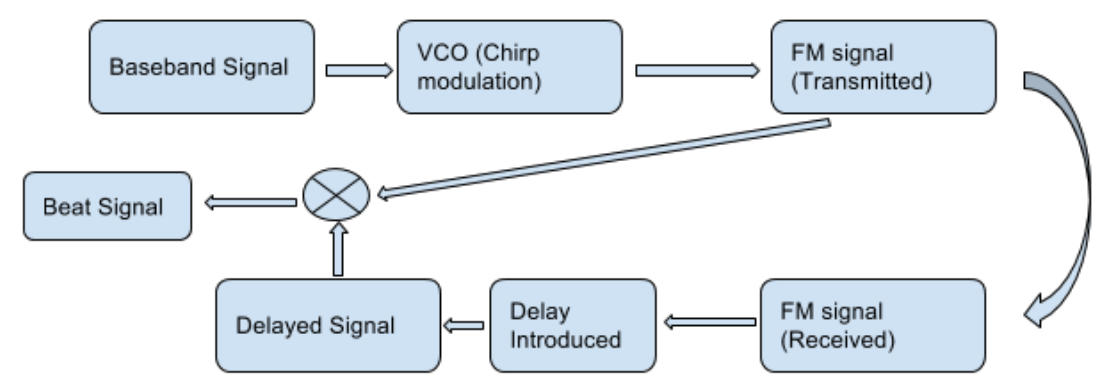

Figure 3.9: System block diagram 


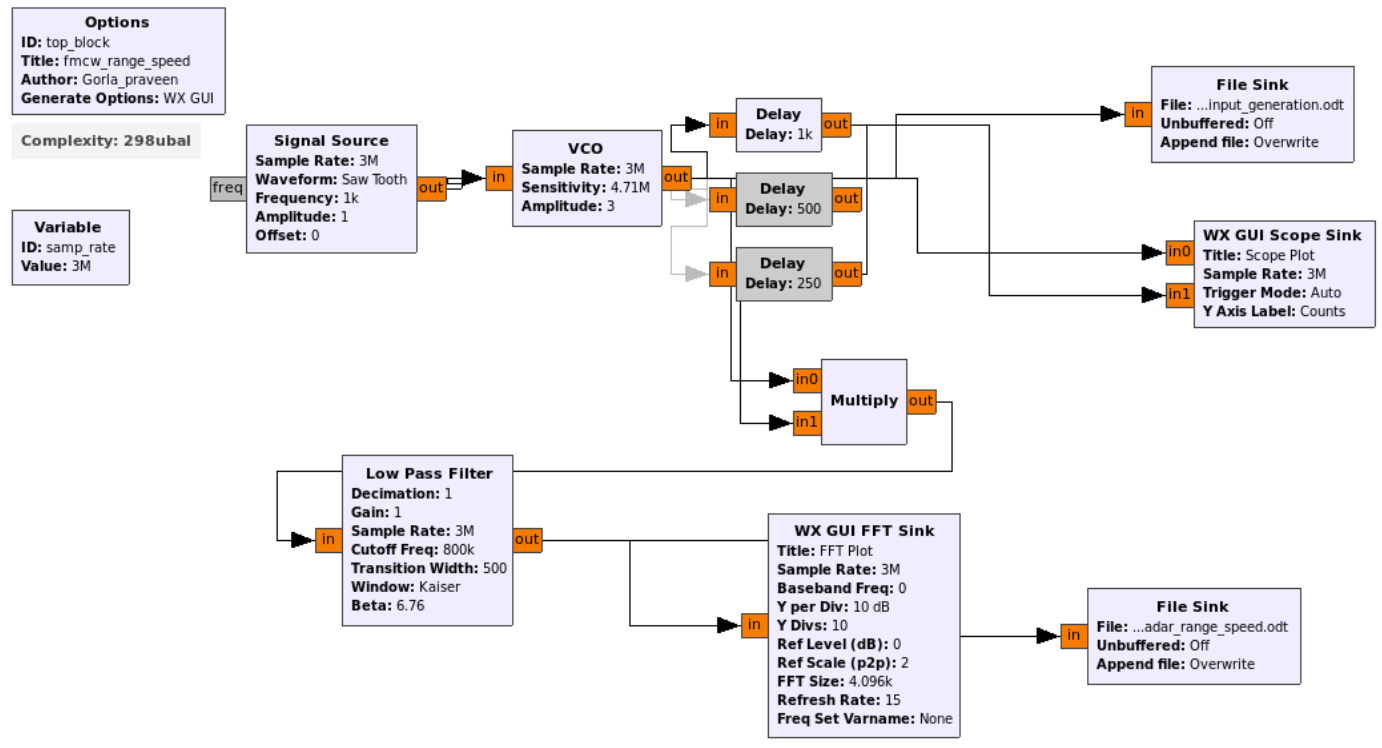

Figure 3.10: GNU Radio Implementation

Assuming single target, a delay of 1000, 500, 250 and 100 samples are introduced respectively to study the scenario at varies TOF intervals. As we use 3Mega Samples per second, delay of 1000 , 500,250 and 100 samples experiences a delay of $0.33 \mathrm{~ms}, 0.16 \mathrm{~ms}, 0.08 \mathrm{~ms}$ and $0.033 \mathrm{~ms}$ respectively which can be treated as $\operatorname{TOF}(\Delta T)$ of signal (creating the virtual delay in receiving a signal ).

Note: Here in GNUradio, delay is introduced in terms of number of samples.

Equations (3.1) and (3.2) is used to determine the range of the target both theoretically and practically respectively. In (3.1) and (3.2) $R$ is range of target from the system, $T_{m}$ is the time period of modulating signal, $\mathrm{B}$ is the bandwidth of the chirp signal, $c$ is velocity of light, $\Delta T$ is delay in the received signal and $f_{b}$ is the beat frequency between transmitted signal and received signal which can be determined from the graph as shown in figures 3.11, 3.12, 3.13 and 3.14.

$$
\begin{aligned}
& R(\text { theoretical range })=\frac{c \cdot \Delta T}{2} \\
& R(\text { practical range })=\frac{c \cdot T_{m} \cdot f_{b}}{2 \cdot B}
\end{aligned}
$$




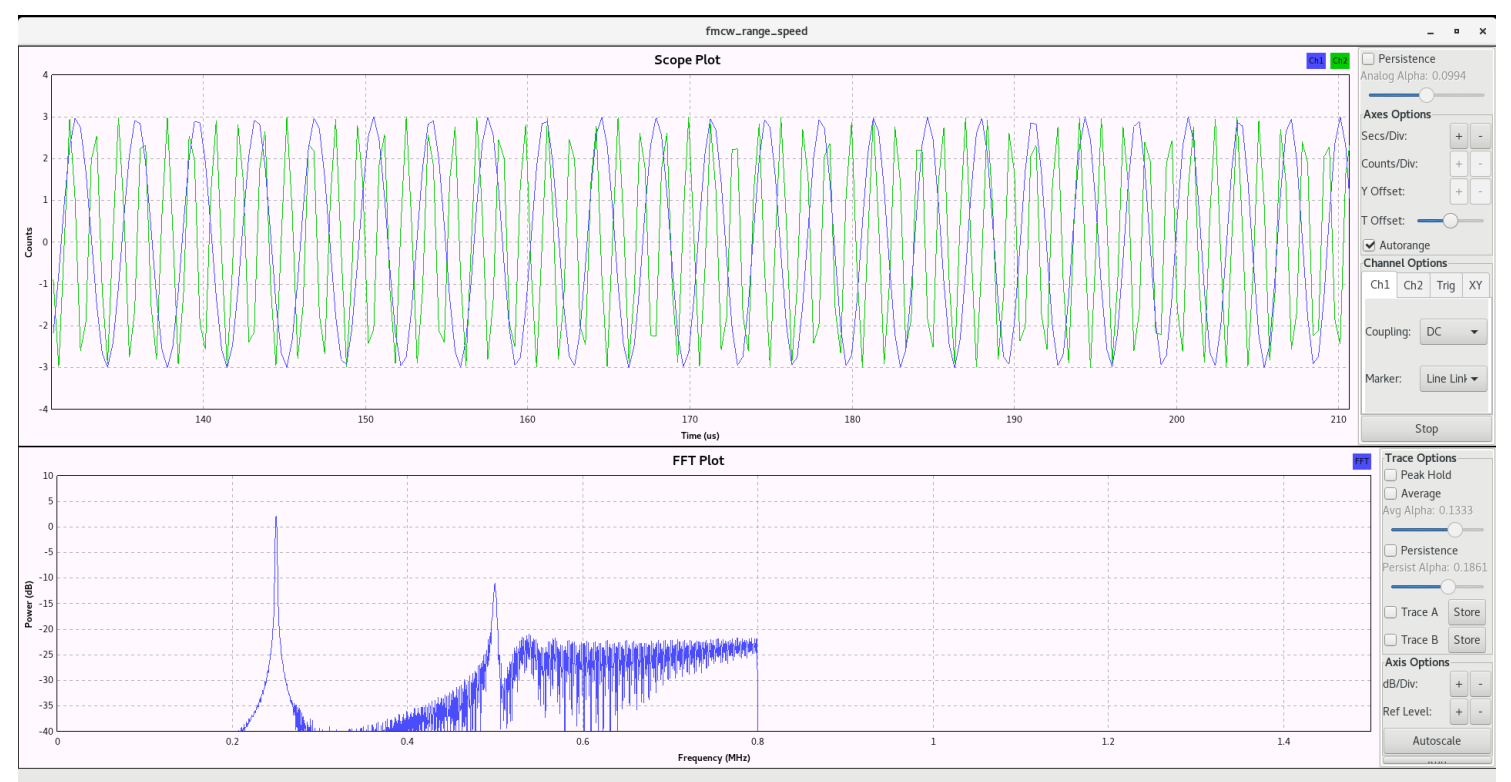

Figure 3.11: Target with delay of 1000 samples

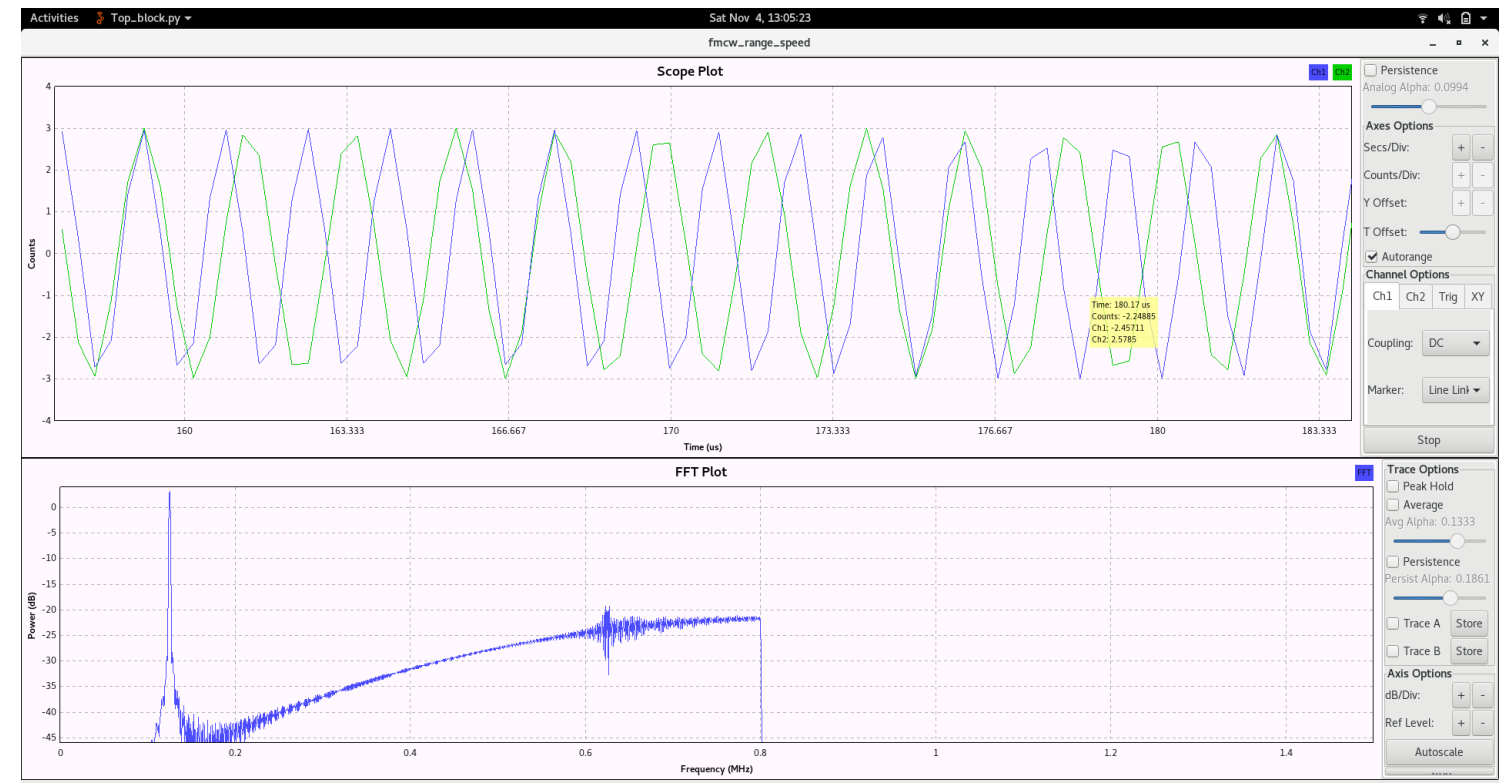

Figure 3.12: Target with delay of 500 samples 


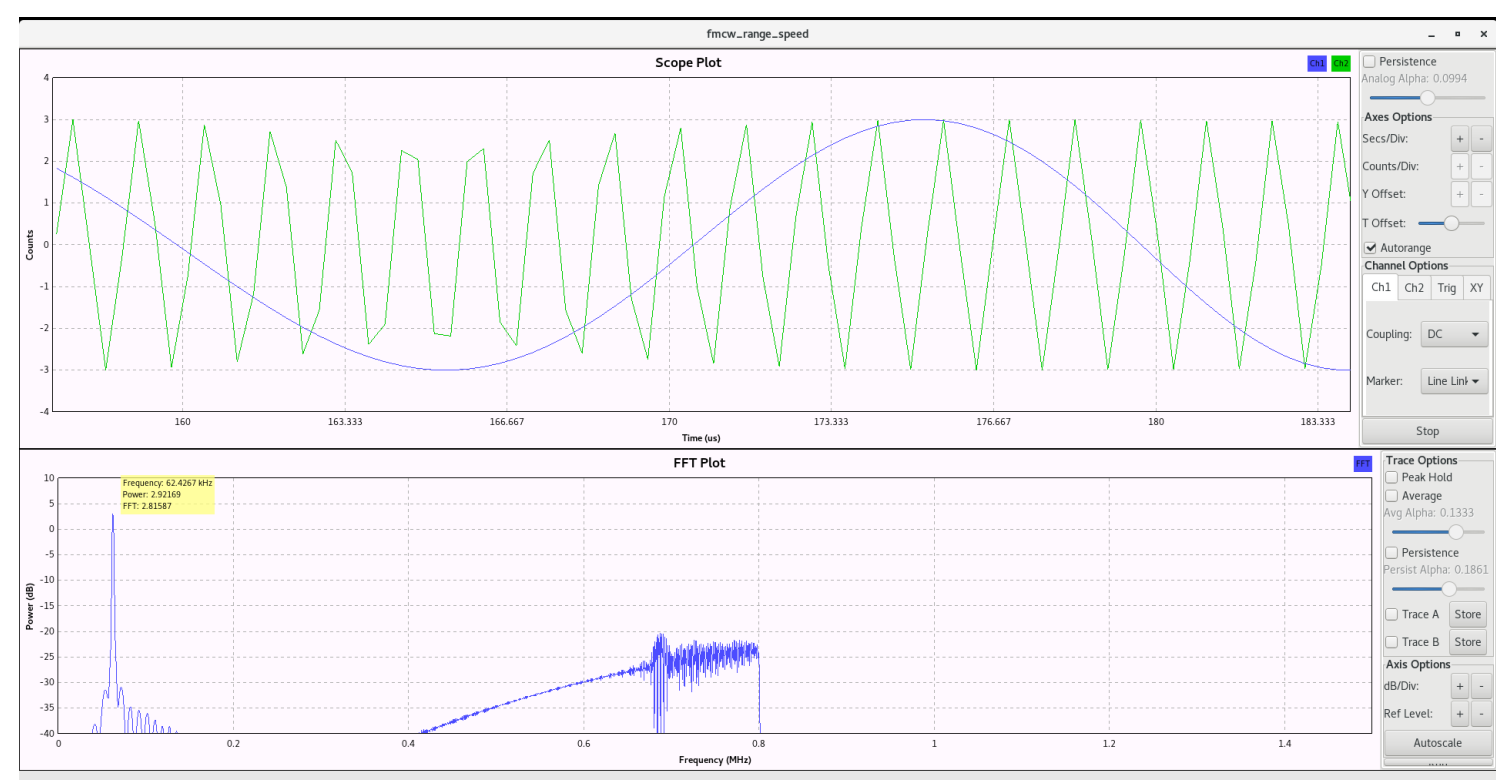

Figure 3.13: Target with delay of 250 samples

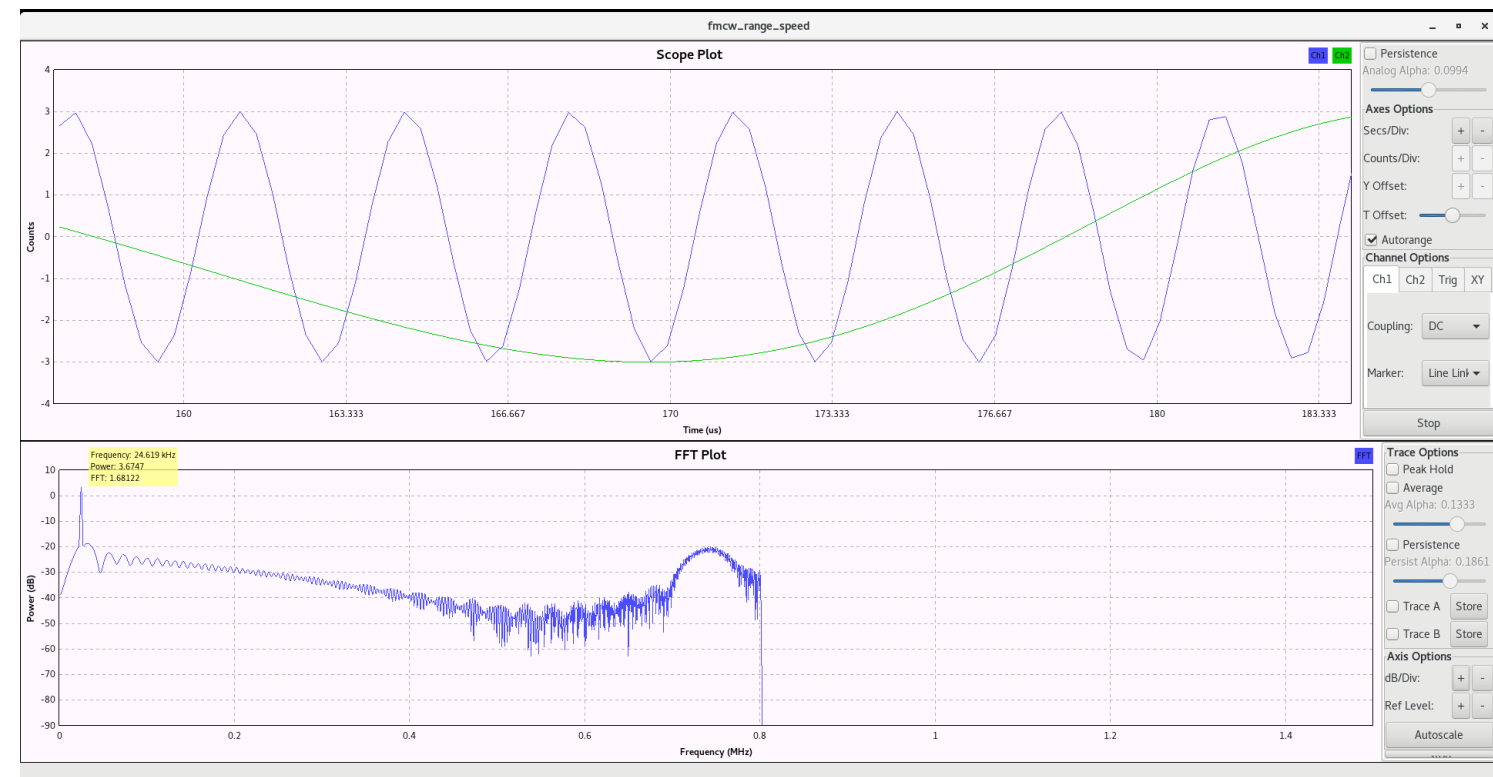

।

Figure 3.14: Target with delay of 100 samples

Observed beat frequencies $f_{b}$ from graphs as shown in figure 3.11, 3.12, 3.13 and 3.14. are $250.88 \mathrm{kHz}, 124.12 \mathrm{kHz}$, and $24.619 k \mathrm{~Hz}$ respectively. 
Now, simulation as shown in figure 3.15 is done and the graph is obtained as shown in 3.16 when there is a presence of three targets with 1000,500 and 250 sample delays respectively.

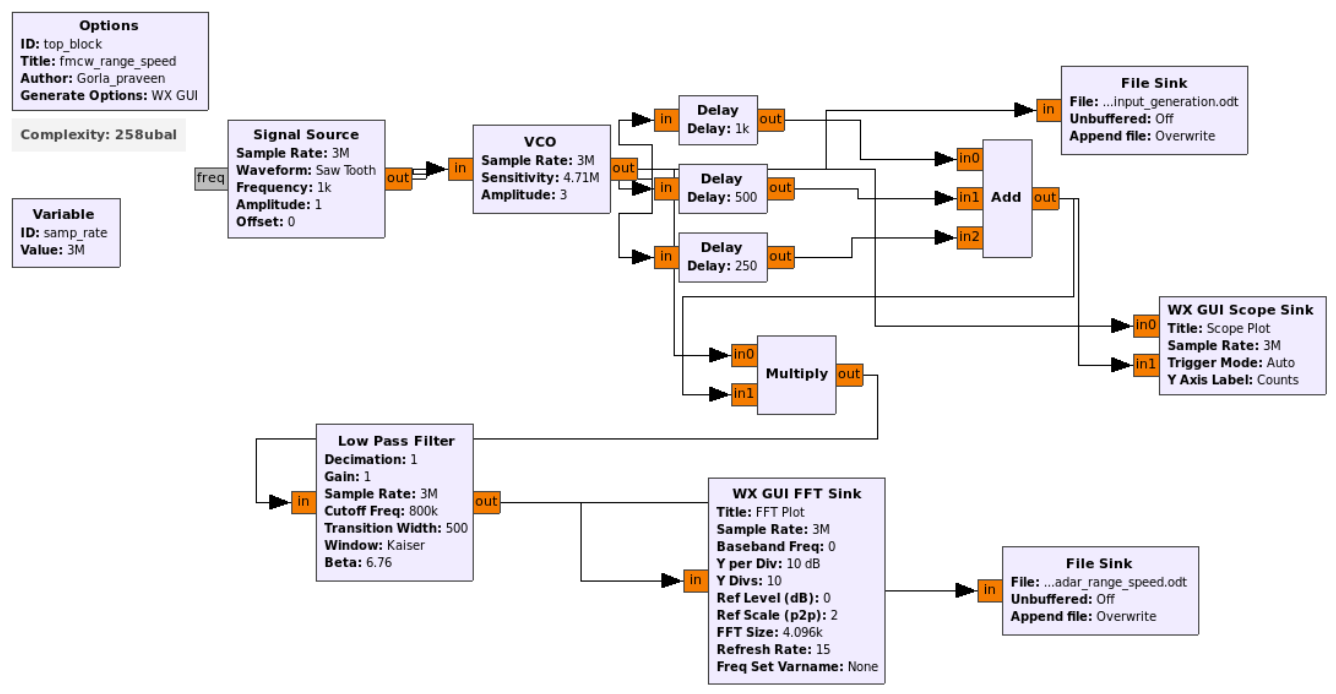

Figure 3.15: GNU Radio implementation for three target detection

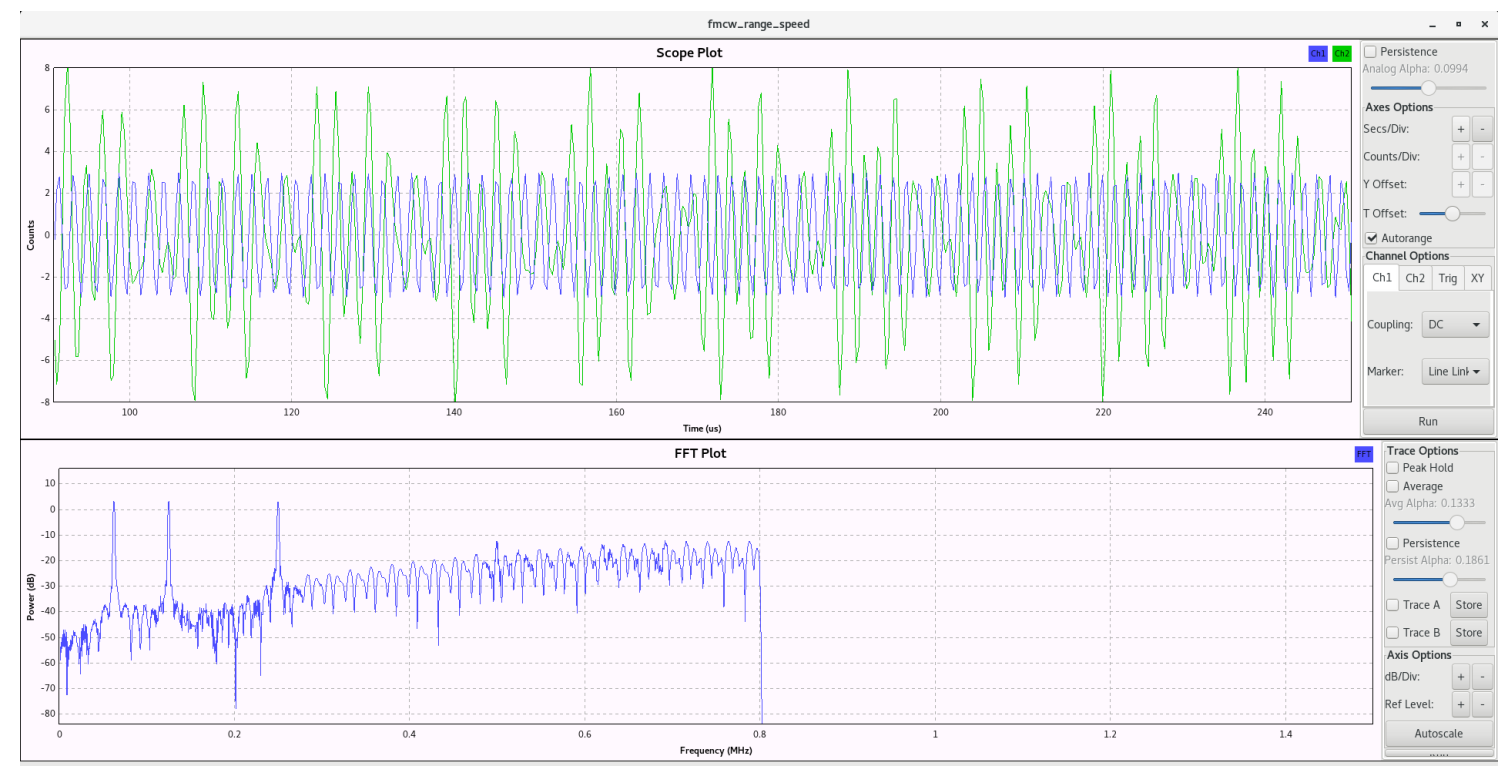

Figure 3.16: Three target presence

In figure 3.16 it is clear that peak values represents beat frequencies $\left(f_{b}\right)$ at $\approx 250 \mathrm{kHz}, 125 \mathrm{kHz}$ and $62.5 \mathrm{kHZ}$ which are almost equal when they treated independently. 
Theoretical and practical ranges are calculated using (3.1) and (3.2) and are represented in 3.9

\begin{tabular}{|c|c|c|c|c|c|}
\hline Step Time & $\begin{array}{l}\text { Theoretical } \\
\text { range }(\mathrm{Km})\end{array}$ & $f_{b}(k H z$ & & \multicolumn{2}{|c|}{$\begin{array}{l}\text { Simulated } \\
\text { range }(\mathrm{Km})\end{array}$} \\
\hline 1000 & 50 & 250.88 & & 50.176 & \\
\hline 500 & 25 & 124.12 & & 24.824 & \\
\hline 250 & 12,5 & 62.4267 & & 12.485 & \\
\hline 100 & 5 & 24,619 & & 4.923 & \\
\hline $1000,500,250$ & $50,25,12.5$ & $\begin{array}{l}250.90, \\
63.3803\end{array}$ & 124.12, & $\begin{array}{l}50.180 \\
12.676\end{array}$ & 24.824 \\
\hline
\end{tabular}

Table 3.9: Theoritical and simulated values

\section{Conclusion on time delay analysis}

Theoretical range and practical simulated range values of targets are found to be equal stating that FMCW signals are capable of detection of about and its range when they are present at longer distances. 


\section{Chapter 4}

\section{Conclusion}

Recent studies have shown that wireless signals have paved for designing low range radar systems in high clutter and low clutter environments with acceptable accuracy. These radar systems are used in indoor for detection of an object, especially for short-range detection. Investigation shows target location can be estimated from the CSI information which inter CSI information can also be traced by Physical Layer Convergence Procedure (PLCP)(Stanislaw Rzewuski et ai.,)

Research scholars have involved in developing capturing the Human Figure through a Wall (Adib et ai., ), Multi human tracking and 3D body shape forming (Adib et al.; ) systems using Frequency Modulated Continuous Wave (FMCW) ( Dijk et al.,) radars which works with a sweep frequency in Ultra Wide Band $(3.1 \mathrm{GHz}-10.6 \mathrm{GHz})$ range.These systems are able to form full body image upon receiving reflections from the body at different frequencies transmitted by the FMCW transmitter.

Further scope of research lies in designing a system which tracks objects and traces the 3D shape of an object using minimum networking devices such as the router(s) with an open architecture which works at central frequencies of $2.4 \mathrm{GHz}(802.11 \mathrm{n})$ and $5 \mathrm{GHz}(802.11 \mathrm{n})$ with a bandwidth of $20 \mathrm{Mhz}$ and 40Mhz respectively. 


\section{References}

Adib, Mao, Kabelac, Katabi and Miller. (2015) Smart Homes that Monitor Breathing and Heart Rate. ACM.

Adib and Katabi. (2013). See Through Walls with Wi-Fi!. ACM.

Adib, Kabelac, Katabi and Miller. (2014) 3D Tracking via Body Radio Reflections. MIT.

Adib, Kabelac, Katabi and Miller. (2014) Multi-Person Localization via RF Body Reflections. MIT.

Ahmed, Talukder and Monjur. (2008). WiFi Radar: Design and Implementation of an Infrastructureless Location Tracking System for Passive Environment. IEEE.

Chetty, Smith, Guo and Woodbridge. (2009). Target Detection in High Clutter using Passive Bistatic WiFi Radar. IEEE.

Chetty, Smith and Woodbridge. (2011). Through-the-wall Sensing of Personnel Using Passive Bistatic WiFi Radar at Standoff Distances. IEEE.

Falcone, Colone, Macera and Lombardo. (2012). Localisation and tracking of moving targets with WiFi-based passive radar. IEEE.

Falcone, Colone, Bongioanni and Lombardo. (2010). Experimental Results for OFDM WiFi-Based Passive Bistatic Radar. IEEE.

Guo, Woodbridge, and C. J. Baker. (2008). Evaluation of Wifi Becon transmission for wireless based passive radar. IEEE.

Rzewuski and Kulpa. (2011). System Concept of WIFI Based Passive radar. INTL JOURNAL OF ELECTRONICS AND TELECOMMUNICATIONS.

Van Dijik, Van der Houwen and Maas. (2008). Multi-Mode FMCW Radar Array with Independent Digital Beam Steering for Transmit and Receive. EuMA.

Wu, Yang, Zhou, Liu and Cao. (2015). Non-Invasive Detection of Moving and Stationary Human with WiFi.IEEE.

Zhou, Wu, Yang, and Liu. (2015). Sensorless Sensing with WiFi. TSINGHUA SCIENCE AND TECHNOLOGY. 


\section{Appendix A}

\section{MATLAB Code}

"Generating. DSSS . / . DBPSK. Modulation".

\%--'DSSS. --Direct. Sequence.. Spread...Spectrum .. Generation .. with .. Barker.

function [dbpsk_sig] = DSSS_DBPSK_Barker

$\mathrm{clc}$

clear

\%."Generating. the . bit . pattern with each bit .28.. samples ..long.".

barker $=\left[\begin{array}{llllllllllll}1 & -1 & 1 & 1 & -1 & 1 & 1 & 1 & -1 & -1 & -1\end{array}\right]$;\% bits .

pattern for $_{-}$dss $=[] ; \%$ pat..

for $\mathrm{k}=1: 11 \%$ sample introducing..

if barker $(1, k)==-1$

signal=-ones $(1,28)$; \%sample introducing..

else

signal=ones $(1,28) ; \%$ sample introducing . .

end

end

pattern_for_dss =[pattern_for_dss signal ];

subplot $(4,1,1) \%$ subplotting..

plot ( pattern for_dss);\% plotting..

axis $\left(\left[\begin{array}{llll}-1 & 308 & -1.5 & 1.5\end{array}\right]\right) ; \%$ axis plot..

title ('Original barker Bit Sequence'); $\%$ Naming..

\% "Generating .. the .. pseudo ... random.. bit".

$\%$.. "pattern .. for.. spreading".

$\mathrm{d}=$ round $(\operatorname{rand}(1,121)) ; \% "$ randm gen of bits $" \ldots$

$\mathrm{pn}_{-} \mathrm{seq}=[]$;

carrier $=[]$; \% Carrier_introductiomn.

$\mathrm{t}=[0: 2 * \mathrm{pi} / 3: 2 * \mathrm{pi}] ; \quad \%$ "Creating 5 samples for one cosine".

for $k=1: 77$

if $\mathrm{d}(1, \mathrm{k})==0$ signal=-ones $(1,4)$; \%"Creating samples and ading them ".. else

end signal=ones $(1,4) ; \%$ "Creating samples and ading them "..".

$\mathrm{c}=\cos (\mathrm{t})$;

carrier $=[$ carrier c $]$; 


$$
\mathrm{pn}_{-} \text {seq }=\left[\mathrm{pn}_{-} \text {seq signal }\right]
$$

end

$\%$ ".. Spreadin.g... of .. sequence.".

spreaded_sig = pattern for $\mathrm{dss}_{-} * \mathrm{pn}_{-}$seq ;

subplot $(4,1,2)$

plot ( spreaded_sig)

axis $\left(\left[\begin{array}{llll}-1 & 308 & -1.5 & 1.5\end{array}\right]\right)$;

title ('Spreaded signal');

display ( size ( spreaded_sig));

display ( size ( carrier));

\%" BPSK . Modulation... of ... the ... spreaded .. signal”.

\%"dbpsk_sig=spreaded_sig.*carrier; \% Modulating the signal

$\%$ differential encoding $y[n]=y[n-1]=x[n] "$.

dbpsk_sig $=\bmod \left(\right.$ filter $\left(1,\left[\begin{array}{ll}1 & -1\end{array}\right]\right.$, spreaded_sig $\left.), 2\right) ; \%$.

$\mathrm{dbpsk}_{-}$sig=dbpsk_sig.* carrier; \%.carrier mul...

subplot $(4,1,3)$; \%.subplot_ting..

plot (dbpsk_sig)\% plot_ting ..

axis $\left(\left[\begin{array}{llll}-1 & 308 & -1.5 & 1.5\end{array}\right]\right) ; \%$ Axis_naming.

title ('DBPSK Modulated Signal'); \% Title_naming..

disp ( size (dbpsk_sig));\% disp_lying_sequence..

\%"Plotting .. the ..FFT ... of .. DSSS . signal".

$\mathrm{y}=\operatorname{abs}\left(\mathrm{fft}\left(\mathrm{xcorr}_{(\mathrm{dbpsk} / \mathrm{sig}))}\right)\right.$;

subplot $(4,1,4) \%$.. subplot_ting .

plot $(\mathrm{y} / \max (\mathrm{y})) \%$ Plot $\mathrm{ting}_{\text {. }}$.

xlabel ('Frequency')\% lavbelingx...

ylabel ('PSD')\% labeling - y..

$\%-$ Generating.

DSSS ./DQPSK. Modulation. "\%.

\%" DSSS..--Direct.. Sequence.. Spread.. Spectrum.. Generation .. with.. Barker function [qpsk_sig] = DSSS_DQPSK_Barker

$\mathrm{clc}$

clear

$\mathrm{M}=4$;

$\%$ "Generating the bit pattern with each bit 28 samples long".

barker $=\left[\begin{array}{lllllllllll}1 & -1 & 1 & 1 & -1 & 1 & 1 & 1 & -1 & -1 & -1\end{array}\right]$; \%bar_kEr_bits $\ldots$

pattern_for_dss $=[]$;

for $\mathrm{k}=1: 11$ 


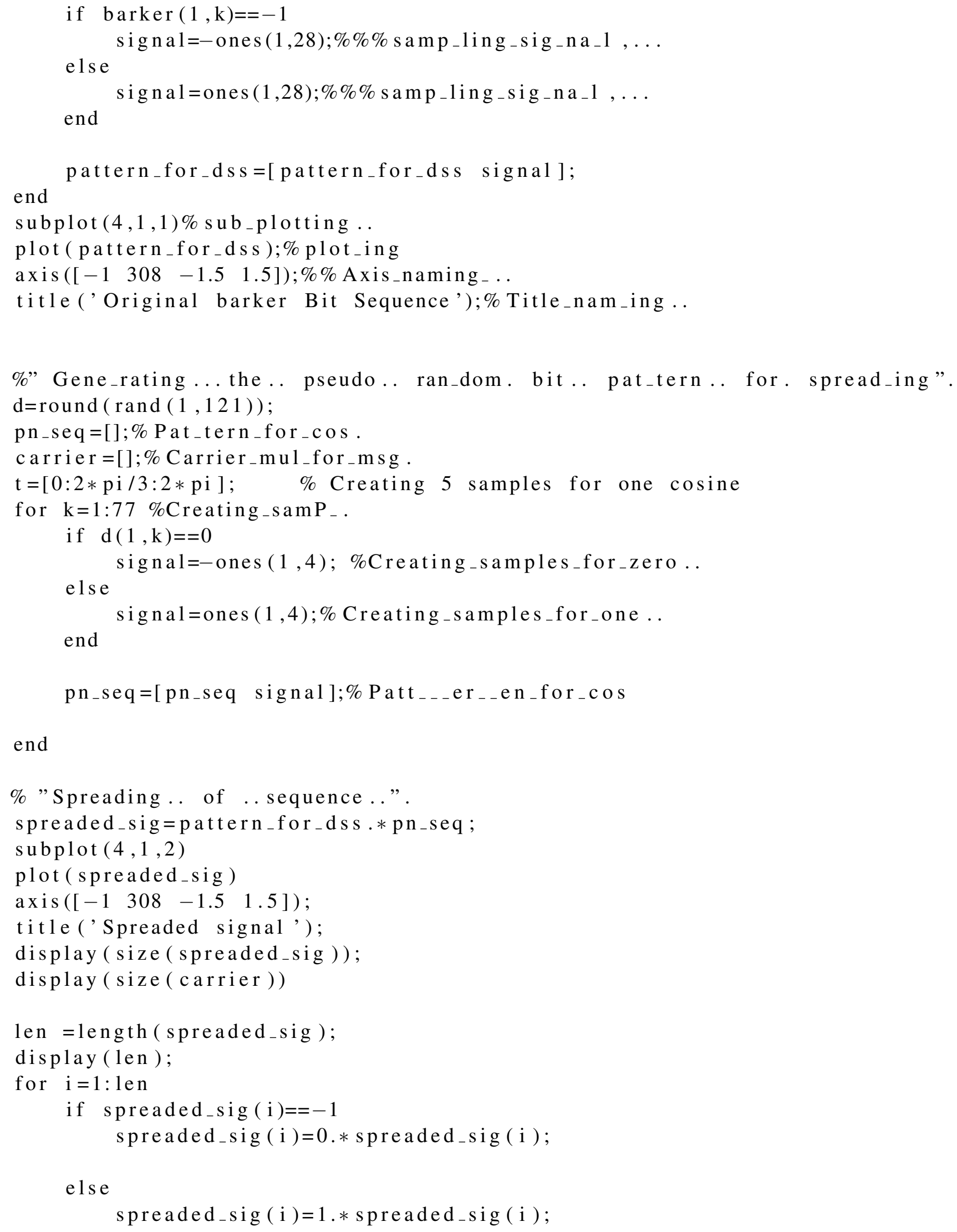




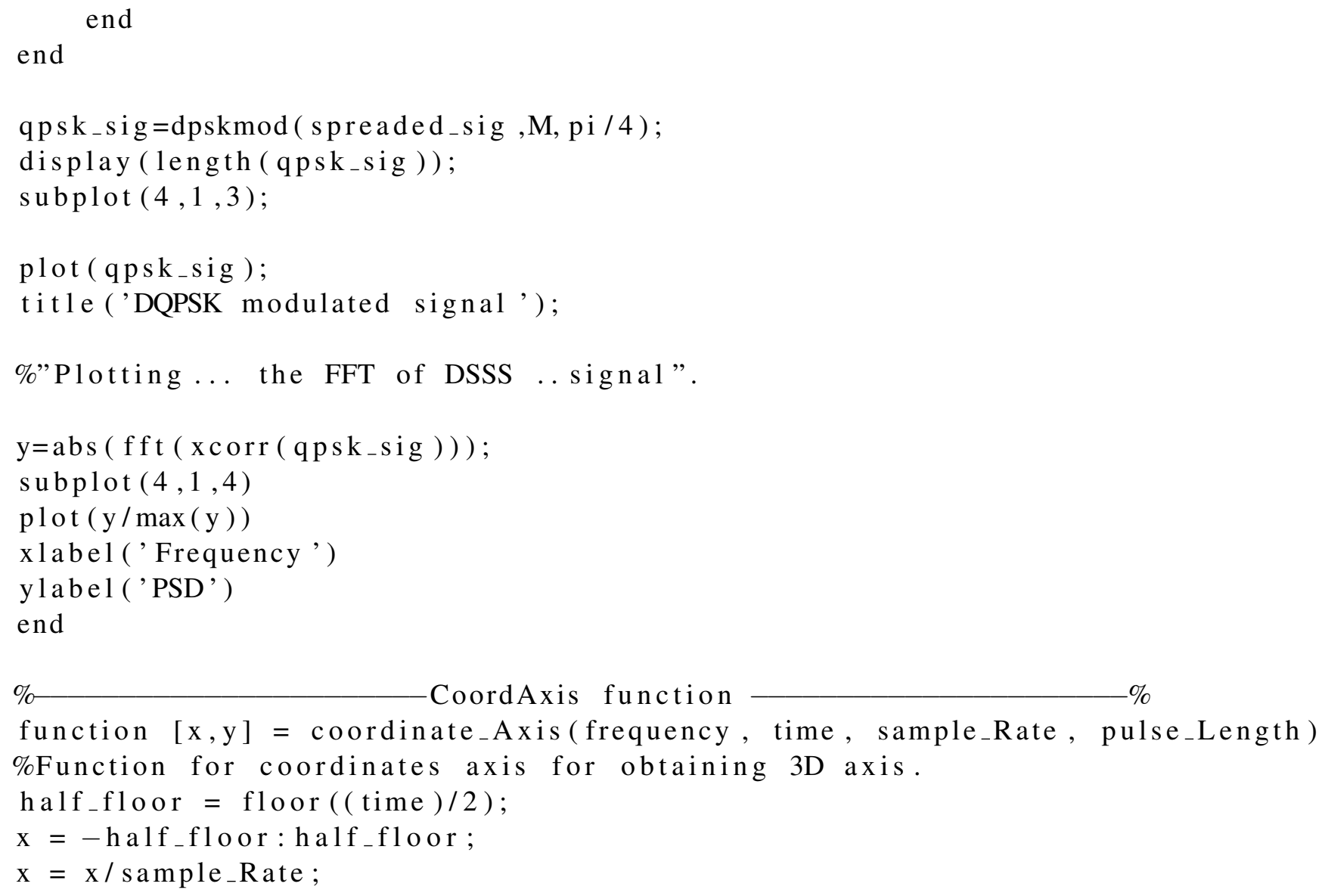

$\%$ ambiguity_function $\%$

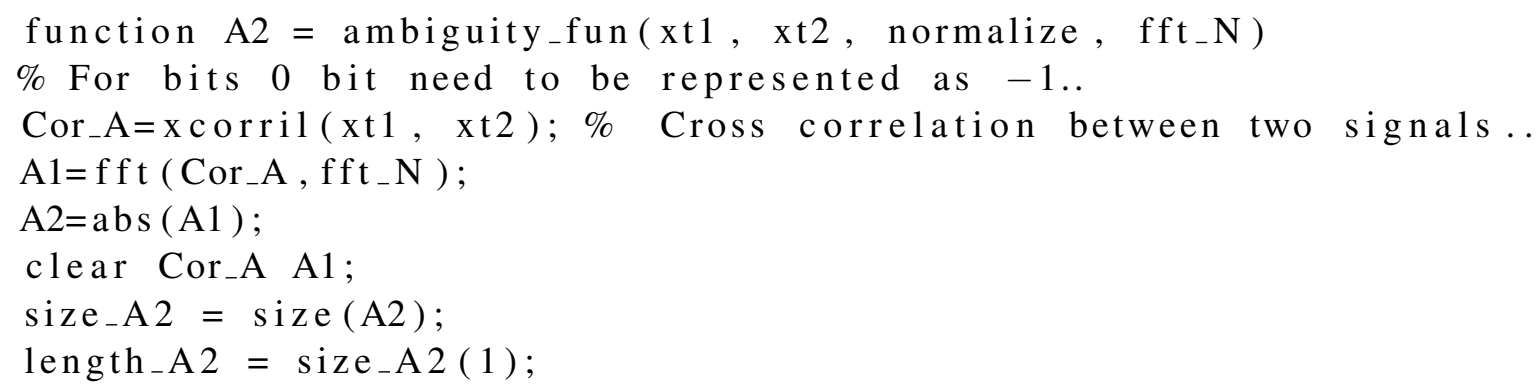




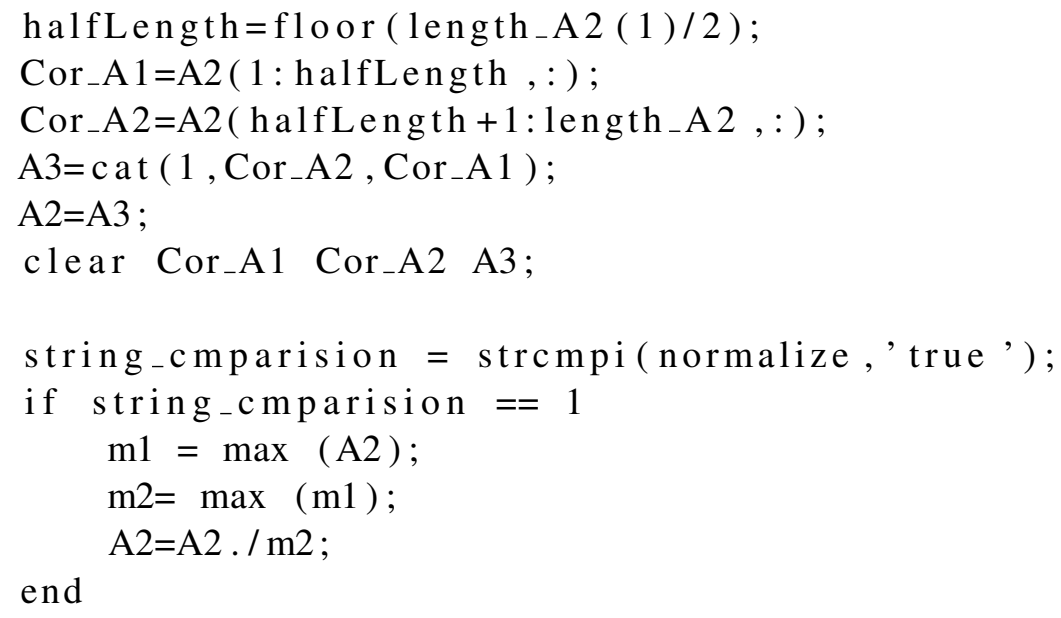

clc;

clear ;

close all;

normalize $=$ true ;

$\mathrm{fftPoint}=256$;

$\left[\mathrm{dbpsk} \_\right.$sig $]=$DSSS_DBPSK_Barker;

Z12=ambiguity_fun(dbpsk_sig, dbpsk_sig, normalize, fftPoint); ambigSize $=$ size $($ Z12);

pulseLength=length ( dbpsk_sig);

$[\mathrm{x}, \mathrm{y}]=\mathrm{coordinate}_{-}$Axis (fftPoint, ambigSize (2), '1', pulseLength);

display (Z12);

display ( ambigSize);

figure ;

$\operatorname{mesh}(\mathrm{x}, \mathrm{y}, \mathrm{Z} 12)$;

title ('Ambiguity function');

axes_handle = xlabel('Delay');

set ( axes_handle, 'FontName', 'Symbol');

axes_handle = ylabel('Doppler Shift');

set ( axes_handle, 'FontName', 'Symbol');

axes ${ }^{\prime}$ andle $=$ zlabel $('|c(t, n)| ')$;

set (axes_handle, 'FontName', 'Symbol') ;

$\%$ ambiguity analysis for DSSS/DQPSK- \% 
$\mathrm{clc}$;

clear ;

close all;

normalize $=$ true ;

$\mathrm{fftPoint}=256$;

[qpsk_sig] = DSSS_DQPSK_Barker;

Z12=ambiguity_fun(qpsk_sig, qpsk_sig, normalize, fftPoint);

ambigSize $=$ size $($ Z12 $)$;

pulseLength=length ( qpsk_sig);

$[x, y]=$ coordinate $\_$Axis (fftPoint, ambigSize (2), '1', pulseLength);

display (Z12);

display (ambigSize);

figure ;

$\operatorname{mesh}(\mathrm{x}, \mathrm{y}, \mathrm{Z} 12)$;

title ('Ambiguity function');

axes_handle = xlabel('Delay');

set ( axes_handle, 'FontName', 'Symbol');

axes_handle = ylabel('Doppler Shift');

set (axes_handle, 'FontName', 'Symbol');

axes_handle $=$ zlabel $\left({ }^{\prime}|\mathrm{c}(\mathrm{t}, \mathrm{n})|{ }^{\prime}\right)$;

set ( axes_handle, 'FontName', 'Symbol') ;

$\%$ Power analysis for object detection- $\%$

clc ;

close all;

\%The distance between the Tx antenna (at $2.4 \mathrm{GHz},-10 \mathrm{dBm}$ amp)to..

$\%$ Rx is $30 \mathrm{~cm}$.

$\%$ First the received values are observed when there is.

\%obstacle between Tx.

\%and Rx antennas.

\% Certain values have been observed and avg is done.

rx_no_obs $1=\left[\begin{array}{lllllll}-41.84 & -41.43 & -41.21 & -41.26 & -41.15 & -41.42 & -41.78\end{array}\right]$;

rx_no_obs $2=\left[\begin{array}{lllllll}-43.70 & -43.30 & -43.07 & -43.92 & -43.27 & -44.40 & -43.60\end{array}\right.$

$-43.83-43.76-44.15-43.88]$;

rx_no_obs $3=\left[\begin{array}{lllllll}-44.59 & -43.57 & -44.99 & -43.64 & -44.34 & -45.04 & -45.21\end{array}\right.$

$\left.\begin{array}{lll}-43.61 & -44.05 & -43.89\end{array}\right]$;

len $1=\operatorname{length}($ rx_no_obs 1$)$;

len $2=1$ ength ( rx_no_obs 2$)$;

len $3=$ length ( rx_no_obs 3$)$;

rx_no_obs1_sum = -290.09;

rx_no_obs2_sum = -480.58;

rx_no_obs3_sum =-442.92; 


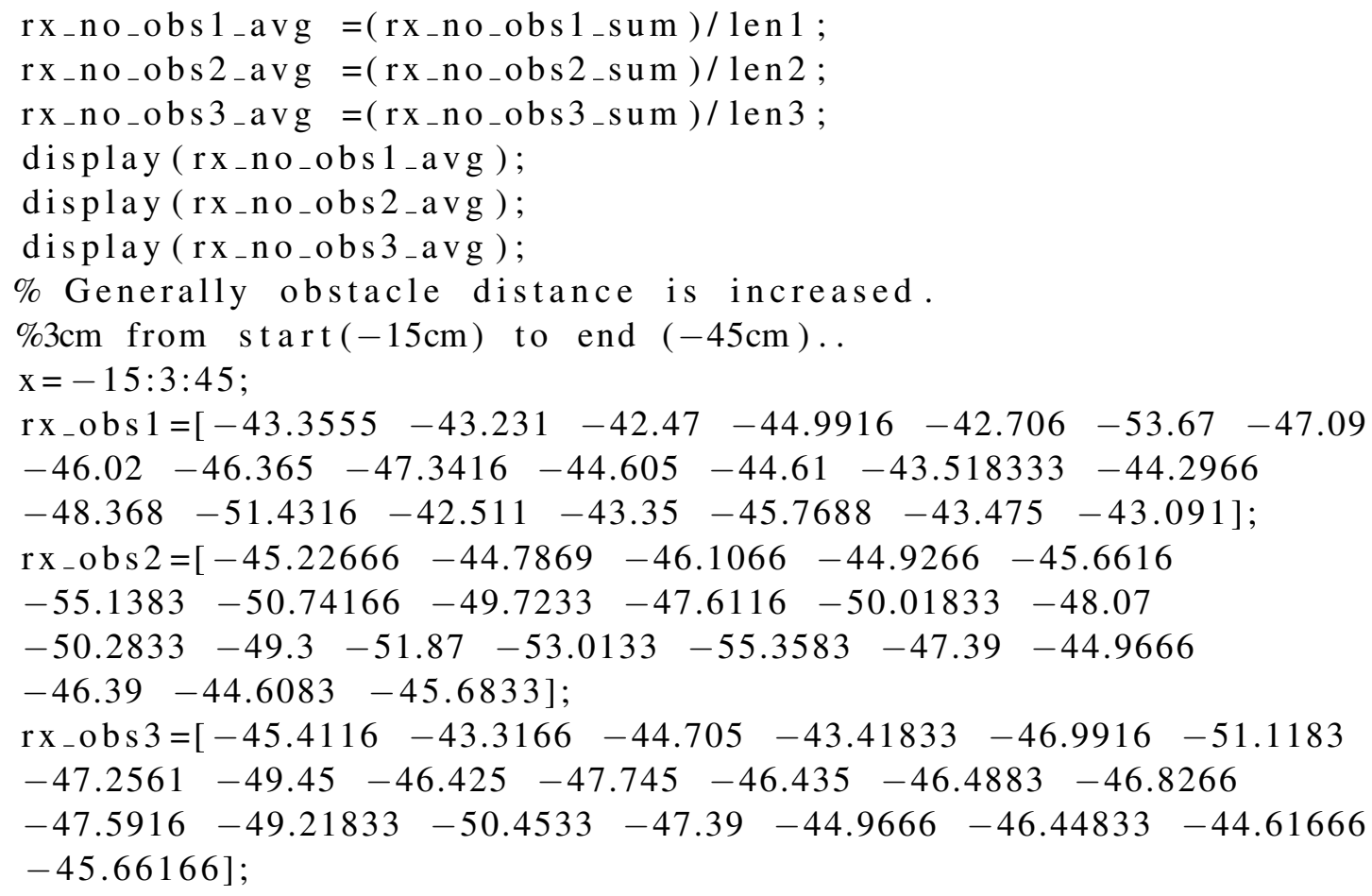

figure ;

semilogy (x, rx_no_obs1_avg,' ks' ');

hold on;

semilogy ( $\mathrm{x}, \mathrm{rx} \mathrm{x}_{-} \mathrm{obs} 1$, $\left.^{\left.\mathrm{o}-{ }^{\prime}\right)}\right)$;

axis $\left(\left[\begin{array}{llll}-20 & +50 & -54 & -40\end{array}\right]\right)$;

grid on ;

legend('Avg $\mathrm{Rx}_{-} \mathrm{dBm}$ when no obstracle is placed',

'Rx_dbm_received by receiver when obstracle moves from Tx to Rx'); xlabel('Distnce (in $\mathrm{cm}, 30 \mathrm{~cm} \mathrm{tx}-\mathrm{Rx}$ ditance) when Obstracle moves from $T x$ to $R x$ taken for every $3 \mathrm{~cm}$ from $-15 \mathrm{~cm}$ to $45 \mathrm{~cm}$ ') ylabel('Received Rx_dBm '); title ('1 st experiments - When obstracle is placed at

different distances from Tx and $\left.\operatorname{Rx}(\operatorname{LOS})^{\prime}\right)$;

figure ;

hold on ;

semilogy (x, rx_no_obs2_avg, 'ks' );

semilogy ( $\left.\mathrm{x}, \mathrm{rx} \mathrm{x}_{-} \mathrm{obs} 2, \mathrm{e}^{-} \mathrm{o}-{ }^{\prime}\right)$;

axis $\left(\left[\begin{array}{llll}-20 & +50 & -54 & -40\end{array}\right]\right)$;

grid on ;

legend('Avg Rx $x_{-} d B m$ when no obstracle is placed',

'Rx_dbm_received by receiver when obstracle moves from $T x$ to $R x$ ') xlabel('Distnce (in $\mathrm{cm}, 30 \mathrm{~cm} \mathrm{tx}-\mathrm{Rx}$ ditance) when Obstracle moves from $T x$ to $R x$ taken for every $3 \mathrm{~cm}$ from $-15 \mathrm{~cm}$ to $45 \mathrm{~cm}$ ') ylabel('Received Rx_dBm'); 
title ('2nd time experiment is conducted - When obstracle is placed at different distances from Tx and Rx (LOS)');

figure ;

semilogy (x, rx_no_obs3_avg, ' ks' ');

hold on;

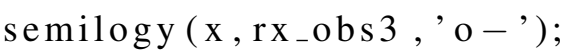

axis $\left(\left[\begin{array}{llll}-20 & +50 & -54 & -40\end{array}\right]\right)$;

grid on;

legend('Avg $\mathrm{Rx}_{-} \mathrm{dBm}$ when no obstracle is placed',

'Rx_dbm_received by receiver when obstracle moves from $T x$ to $R x$ ');

xlabel('Distnce (in $\mathrm{cm}, 30 \mathrm{~cm} \mathrm{tx}-\mathrm{Rx}$ ditance) when Obstracle

moves from $\mathrm{Tx}$ to $\mathrm{Rx}$ taken for every $3 \mathrm{~cm}$ from $-15 \mathrm{~cm}$ to $45 \mathrm{~cm}$ ')

ylabel('Received Rx_dBm ');

title ('3rd time experiment is conducted - When obstracle is

placed at different distances from Tx and Rx (LOS)');

hold off;

$\%-$

End of Matlab

Codes" 